\title{
Gradhiva
}

GRADHIV

Revue d'anthropologie et d'histoire des arts

16 | 2012

Chines, l'État au musée

\section{Yingti/ruanti (hardware/software). La création d'un hall culturel hakka à Taïwan}

Yingti/Ruanti (Hardware/Software). The Creation of a Hakka Culture Hall in Taiwan

\section{Anne-Christine Trémon}

\section{OpenEdition}

\section{Journals}

Édition électronique

URL : http://journals.openedition.org/gradhiva/2489

DOI : $10.4000 /$ gradhiva.2489

ISSN : 1760-849X

\section{Éditeur}

Musée du quai Branly Jacques Chirac

Édition imprimée

Date de publication : 1 décembre 2012

Pagination : 130-155

ISBN : 978-2-35744-048-7

ISSN : 0764-8928

\section{Référence électronique}

Anne-Christine Trémon, «Yingti/ruanti (hardware/software). La création d'un hall culturel hakka à

Taïwan », Gradhiva [En ligne], 16 | 2012, mis en ligne le 01 décembre 2015, consulté le 03 mai 2019.

URL : http://journals.openedition.org/gradhiva/2489 ; DOI : 10.4000/gradhiva.2489 


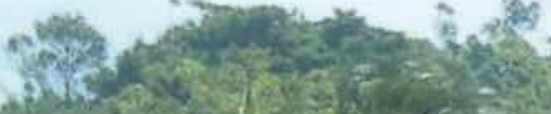

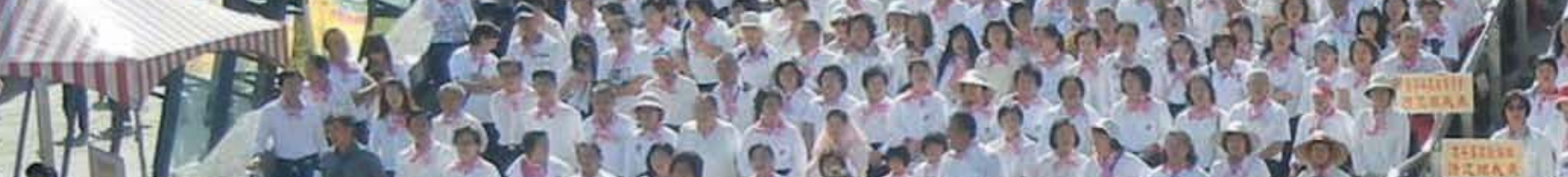

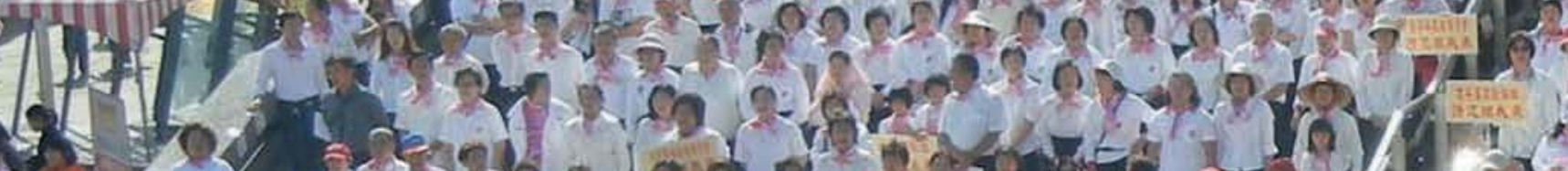

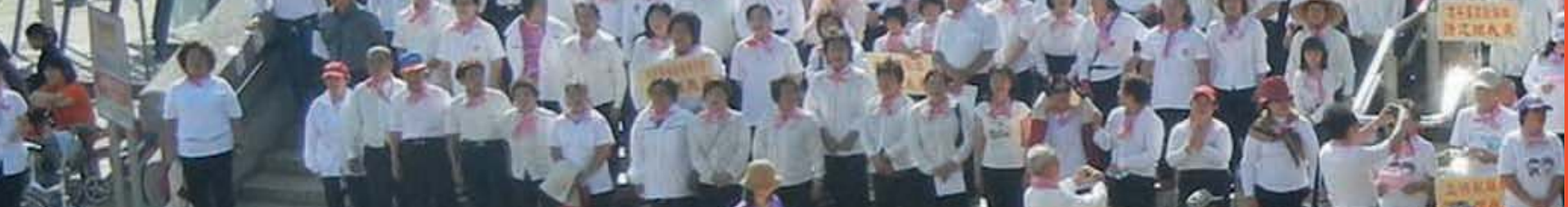

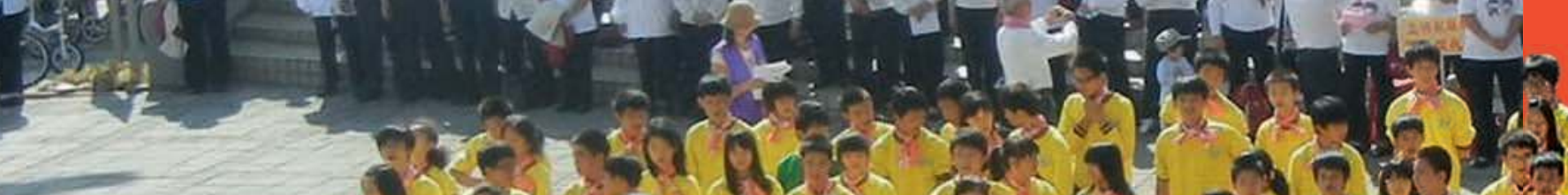

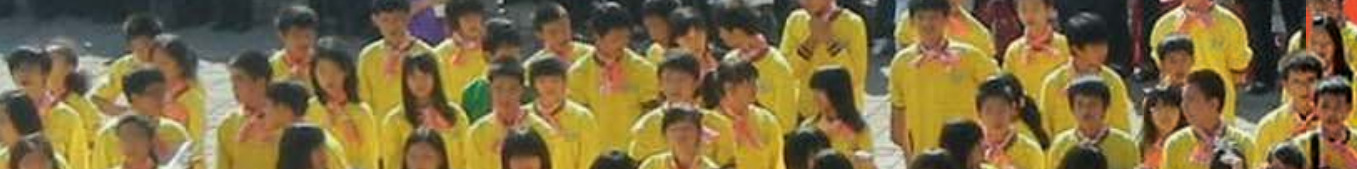

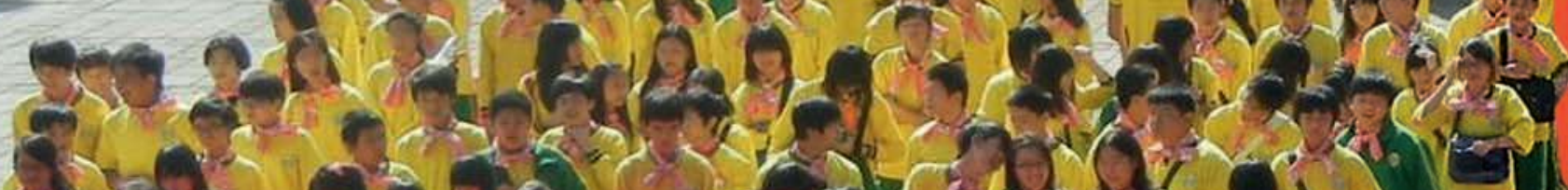
(5)

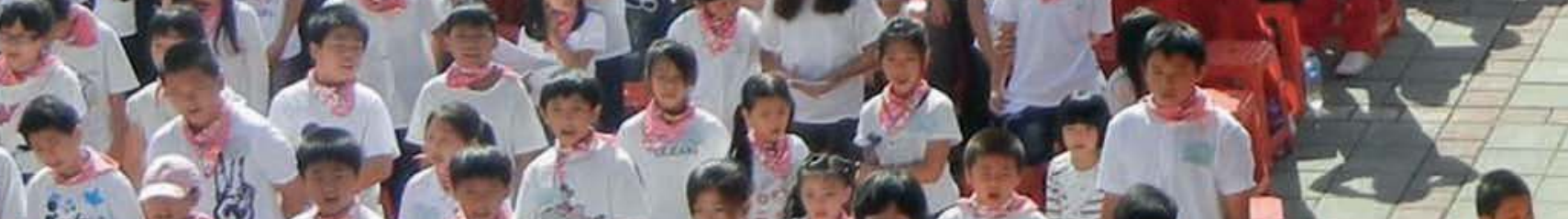

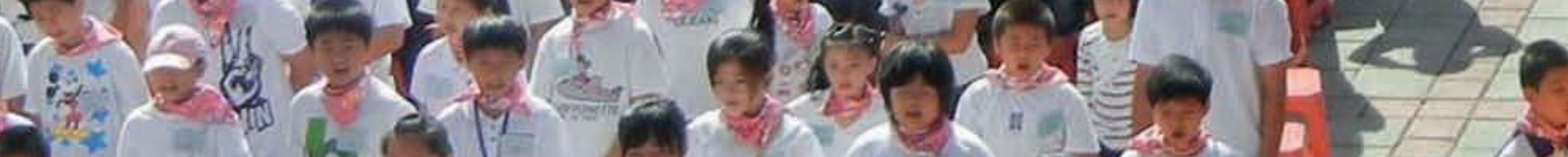

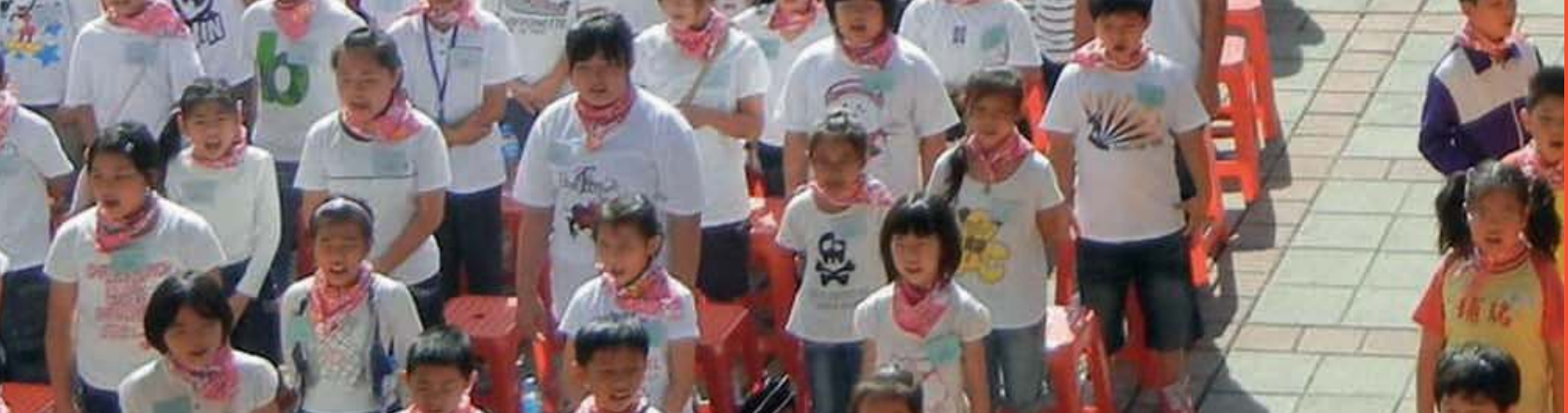




\section{Yingti/ruanti (hardware/software)}

La création d'un hall culturel hakka à Taïwan

\section{par Anne-Christine Trémon}

Dans le cadre de la politique multiculturelle de protection des minorités menée par le gouvernement taïwanais, le Comité ministériel aux affaires hakkas (Keweihui) a planifié dix-huit «halls culturels hakkas » dans chaque comté et municipalité. L'article retrace le processus de création d'un de ces halls dans le nord de l'île de Taïwan au cours de la décennie 2000. L'aménagement du hall procède à l'incorporation d'artistes locaux dans une tradition nationale hakka réinventée. Il est montré comment sa genèse s'est produite à l'intersection d'une politique impulsée par le haut et d'un projet émanant d'acteurs locaux. L'article met en évidence comment les catégories yingti (hardware) et ruanti (software) président à sa fabrication, et impliquent une opposition et une complémentarité entre «contenant » et «contenu», «État » et «société», qui agissent à toutes les étapes de sa mise en œuvre. 


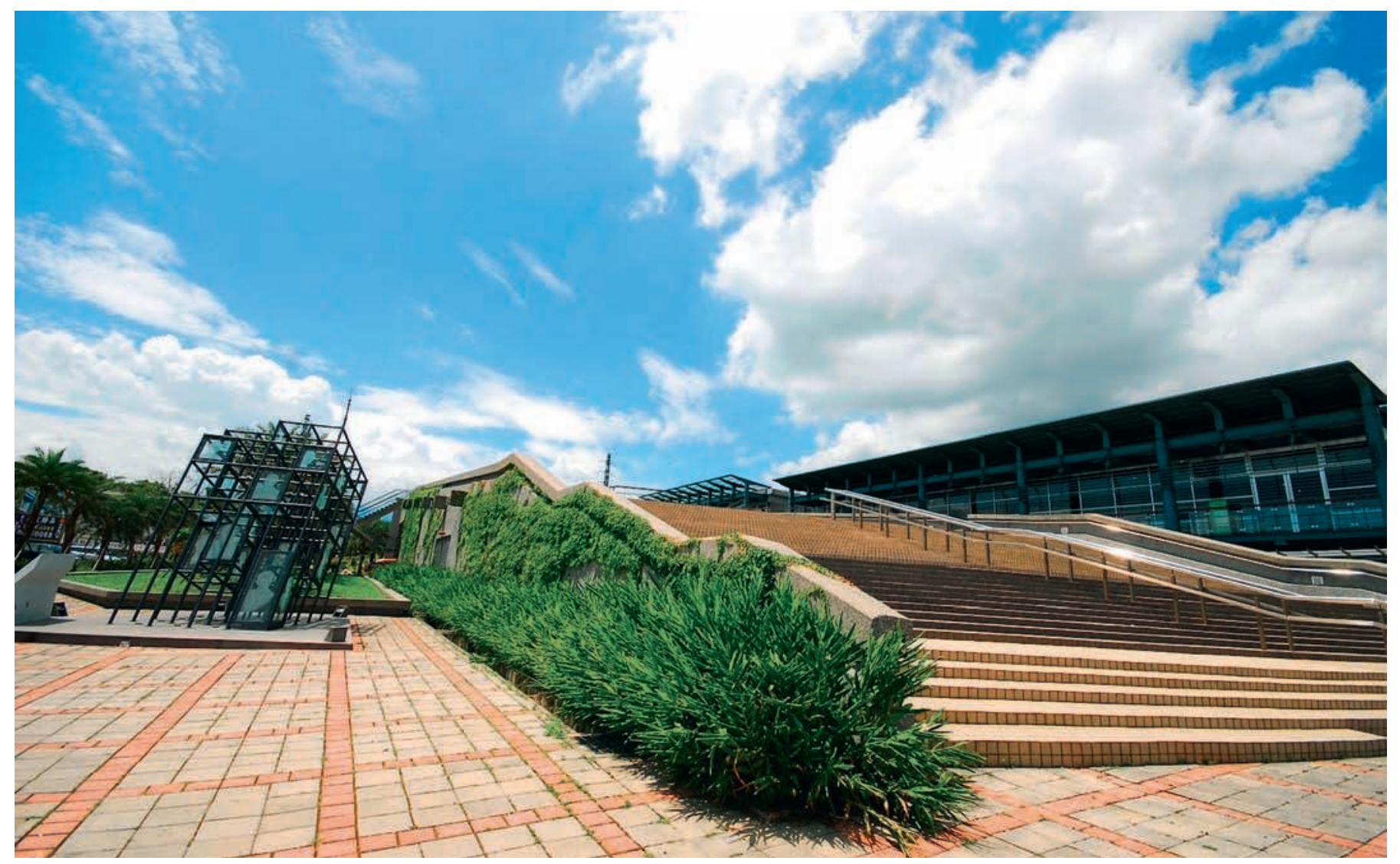

fig. 1

Vue extérieure du bâtiment du hall culturel hakka de Longtan (Taoyuan) (c) Bureau des affaires hakkas du gouvernement du district de Taoyuan.
Longtan ${ }^{1}$, l'étang du Dragon, doit son nom au bassin autour duquel ce bourg s'est édifié après l'apparition d'un dragon jaune surgi des eaux. Intimement lié à l'élément aquatique, le dragon est associé, dans la civilisation chinoise, à la fertilité agricole et au pouvoir impérial. Aussi le nom même de «Longtan » est-il emblématique de l'histoire de la colonisation han de Taïwan, qui entraîna le défrichage des terres par les immigrants venus du continent chinois aux $X V I^{e}$ et $x V I I I^{e}$ siècles. Originaires des provinces du Fujian et du Guangdong, ceux-ci comprenaient les Hokkiens, les plus nombreux, et les Hakkas (Kejia), minoritaires. Refoulant les aborigènes taïwanais dans les montagnes, les Hakkas, eux-mêmes relégués en périphérie des terres planes du littoral par les Hokkiens, occupèrent le piémont. Dans la moitié nord de l'île, on dénombre quinze kejiazhuang, localités à prédominance hakka, situées à cheval sur les trois xian (districts) de Taoyuan, Xinzhu et Miaoli ${ }^{2}$. Longtan, le kejiazhuang le plus méridional du district de Taoyuan, se situe aux confins de la plaine littorale du nord-ouest de l'île de Taïwan. Sa situation à mi-distance de la capitale, Taipei, et de l'autre grande ville, Hsinzhu, a accéléré, depuis une dizaine d'années, sa transformation de petit bourg rural en ville dont la population s'élève à plus de 100000 habitants. Les collines alentour sont grignotées par les lotissements où viennent s'installer les cols blancs qui travaillent dans les instituts de recherche militaire ou pour l'armée de l'air de la république de Chine.

C'est face aux terrains d'entraînement militaires, à l'extérieur du bourg de Longtan, qu'a été construit en 2005 le «hall culturel hakka du district 
de Taoyuan», Taoyuanxian Kejia Wenhuaguan. Guan désigne un bâtiment public et se retrouve dans les termes musée (bowuguan) et ambassade (dasheguan); il ne s'agit ni d'un «centre» (zhongxin) ni d'un institut (yuan). La recherche d'une traduction fidèle contraint à employer un anglicisme, «hall ». Aménagé sur une parcelle longue et étroite d'environ deux hectares, le hall comprend deux éléments: dans sa moitié nord, un assemblage de bâtiments, et dans sa moitié sud, un parc. Vu de haut, l'ensemble évoque les habitations paysannes en forme de $U$, courantes dans la région. La cour intérieure est semi-ouverte - l'aile gauche étant constituée des bâtiments, l'aile droite d'une passerelle aérienne qui relie le premier étage à un campanile de briques rouges sur lequel est apposée l'inscription en caractères dorés Taoyuanxian Kejia Wenhuaguan. Cette signalétique est identique pour tous les bâtiments relevant des pouvoirs publics.

L'ouverture de ce hall hakka s'inscrit dans un double contexte: celui du «boom» muséal que connaît Taïwan depuis trois décennies et celui d'une «indigénisation» multiculturelle (Corcuff 2002; Lee 2005). Dans l'ère postautoritaire, l'État a recentré son action sur la création et l'affirmation d'une culture nationale taïwanaise, sans cesser cependant d'intervenir fortement dans le domaine de la culture (Winckler 1994: 22). Après l'élection du premier président taïwanais non Guomindang, Chen Shui-bian, du Parti démocratique progressiste (Minjindang ${ }^{3}$ ) en mars 2000, on a vu se renforcer un multiculturalisme (duoyuan wenhua) adossé au principe d'une division de la population en quatre groupes ethniques ${ }^{4}$. Cette politique multiculturelle est passée par l'établissement d'instances représentatives pour les groupes minoritaires, les aborigènes et les Hakkas, et par la création de chaînes de radio et de télévision, de festivals et d'associations culturelles. Le Keweihui, abréviation de xingzhengyuan kejia weiyuanhui (Comité du Yuan exécutif 5 pour les Hakkas), est établi le 14 juin 2001. Ses objectifs principaux sont énoncés dans le plan nommé «Objectif 2008: campagne de développement national pour les nouveaux Hakkas "; à savoir: promouvoir la langue et la culture hakka par le renforcement de l'apprentissage linguistique, encourager les recherches historiques et ethnologiques, mettre en place des sites internet et une chaîne de télévision hakka, et ouvrir des musées et des centres culturels. Dix-huit halls et centres culturels hakkas ont été planifiés par le Keweihui dans chaque district et municipalité de l'île. Ces établissements sont classés en deux niveaux, central ou local, selon qu'ils sont gérés par le centre (zhongyang), c'est-à-dire directement par le Comité des affaires hakkas, ou par les gouvernements de district (xian).

Suivant les conseils de Yang Tsun-rong, professeur au Collège d'études hakkas de l'université centrale de Zhongli, je choisis Longtan pour sa réputation de kejiazhuang «typique» et pour sa proximité avec Taipei, où je résidais ${ }^{6}$. Après avoir flâné en bordure de l'étang, je traversai les ruelles animées du centre jusqu'à l'hôtel de ville (xiang gongsuo), où je m'enquis des prochaines manifestations culturelles. Les employés me proposèrent de monter à l'étage pour y rencontrer le maire de Longtan, qui m'accueillit sans formalités. Je tombais à pic: la cérémonie d'inauguration du hall culturel hakka du xian de Taoyuan, à laquelle il me convia, était imminente. J'entamai alors une enquête qui m'amena à rencontrer l'ensemble des acteurs impliqués dans l'élaboration de ce projet.
1. Les caractères chinois figurant dans ce texte sont non simplifiés, conformément au système d'écriture en vigueur à Taïwan. En revanche, la norme de romanisation adoptée pour les termes chinois est le hanyu pinyin. Bien qu'il ne soit pas très courant à Taïwan, ce procédé de transcription est celui qui a été adopté au sein du hall hakka, notamment pour les noms des artistes qui y sont présentés. Ceux-ci sont souvent romanisés sous une autre forme, et à un même nom correspondent parfois plusieurs romanisations connues. Dans cet article, seule la transcription du nom du musicien Deng Yu-xian respecte celle souhaitée par ses descendants : "Deng Yu Shian »

2. Les districts (xian) et les villes (shi) composent l'échelon intermédiaire entre le centre (zhongyang) et les bourgs et comtés (xiang), à leur tour subdivisés en villages (cun).
3. Le Parti démocratique progressiste, fondé en 1986, un an avant la levée de la loi martiale, fut le premier parti d'opposition autorisé à Taïwan. Depuis le retrait de l'île des troupes nationalistes, celle-ci avait été soumise au régime autoritaire du Guomindang . Le Parti démocratique progressiste rejette l'idée d'une réunification des "deux Chine " et défend le principe d'un État taïwanais indépendant.

\section{Les aborigènes} (yuanzhuminzu), $2 \%$ de la population, les continentaux (waishengren), 13\%, les Hokkiens ou Minnans, $70 \%$, et les Hakkas (kejiaren), environ $15 \%$.

5. L'exécutif du pouvoir taïwanais.

6. J'y étais en qualité de postdoctorante à l'Academia Sinica. Entre novembre 2005 et mars 2006, je fis à raison de deux fois par semaine l'aller-retour entre Taipei et Longtan. Je résidai ensuite à Longtan en juillet et août 2006 , et retournai à Taïwan en décembre 2008 pour observer l'aménagement intérieur et clore mon enquête. 
En retraçant le processus de sa conception, je montrerai en quoi le hall est un projet politique qui vise à incorporer la culture locale tout en y inscrivant la présence de l'État. Le projet de hall, impulsé d'en haut, est révélateur de tensions entre les usages politiques de la culture et les attentes de la société civile locale. C'est ce que dévoilent, en premier lieu, les catégories à travers lesquelles il a été conçu. Les termes yingti et ruanti, traduisibles par hardware et software, témoignent de ce que la politique muséale est pensée comme relevant de la promotion étatique de l'industrie de pointe, au même titre que les secteurs économiques hightech. Ils distinguent des étapes successives de la construction du hall en même temps qu'ils délimitent la division du travail entre les échelons «national» (ou «central») et «local», et agissent comme les révélateurs d'une tentative de séparation entre la politique étatique de construction du «contenant» et la définition locale du «contenu» culturel. L'inauguration du yingti, le hall encore vide, en novembre 2005, précède celle du ruanti, le hall rempli. Celle-ci a été repoussée jusqu'en septembre 2008 en raison de difficultés apparues dans l'intervalle, liées à l'échec de la délégation par le gouvernement local du xian de la gestion du contenu du hall à une entreprise privée.

Cette même tension entre le national et le local sous-tend les choix relatifs à la localisation, l'aménagement intérieur et l'architecture du hall. Conçu autour de deux figures locales originaires de Longtan, l'écrivain Zhong Zhao-zheng et le compositeur Deng Yu Shian, le hall est construit sur un terrain appartenant à l'État central. Je montrerai en quoi l'insertion de ces figures dans le hall et leur mise en série avec d'autres artistes hakkas dans l'espace d'exposition mettent en tension le national et le local en ce qu'elles entendent non seulement transcrire dans la localité même la présence de l'État, mais aussi insérer la culture hakka locale dans un ensemble national. Ces actes d'incorporation empruntent aux modalités cultuelles de la religion chinoise, comme on le verra à travers la manière dont la figure de Deng Yu Shian a été transposée, mais aussi dans l'agencement du hall qui l'apparente à un temple et l'affichage d'une unité culturelle hakka autre que celle, cultuelle, du temple des Yimin situé non loin de Longtan. Le hall constitue ainsi, dans une certaine mesure, une alternative séculaire et étatique à la culture religieuse locale, si vivante à Taïwan - l'un des pays au monde qui compte le plus grand nombre de temples par habitant. Par son inscription dans la grille administrative taïwanaise (un hall hakka étant programmé dans chaque xian ou ville) et son objectif de promouvoir une unité culturelle hakka à l'échelle nationale, le hall opère un double mouvement de «localisation » et de «nationalisation» des figures artistiques qu'il met en exergue. Dans le même temps, les options suivies pour son architecture et son contenu, et les réactions critiques des habitants de Longtan vis-à-vis de ces choix, sont révélatrices du paradoxe auquel est confrontée la politique multiculturelle menée par le gouvernement taïwanais entre la reconnaissance publique donnée à la diversité culturelle et la volonté d'affirmer une unité nationale. Elles soulignent également les tensions entre la politique muséale menée par l'État et la fixation de la culture locale, hakka, dans un hall ou un musée. La distinction yingti/ruanti renvoie, in fine, à la relation de complémentarité antagoniste entre l'État et la société locale qui se joue tout au long du déploiement de ce projet. 


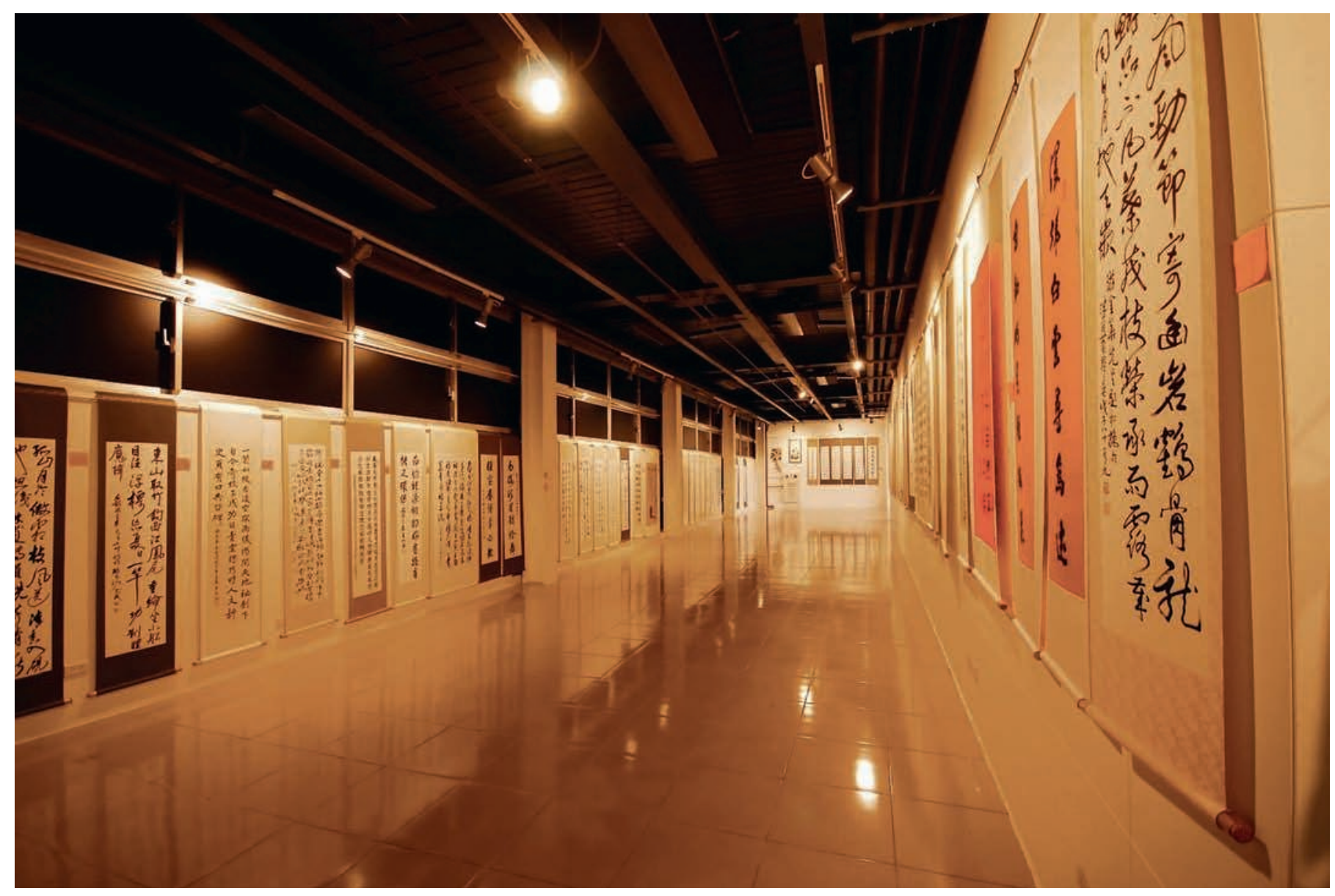

fig. 2

Salle du hall culturel hakka (C) Bureau des affaires hakkas du gouvernement du district de Taoyuan.

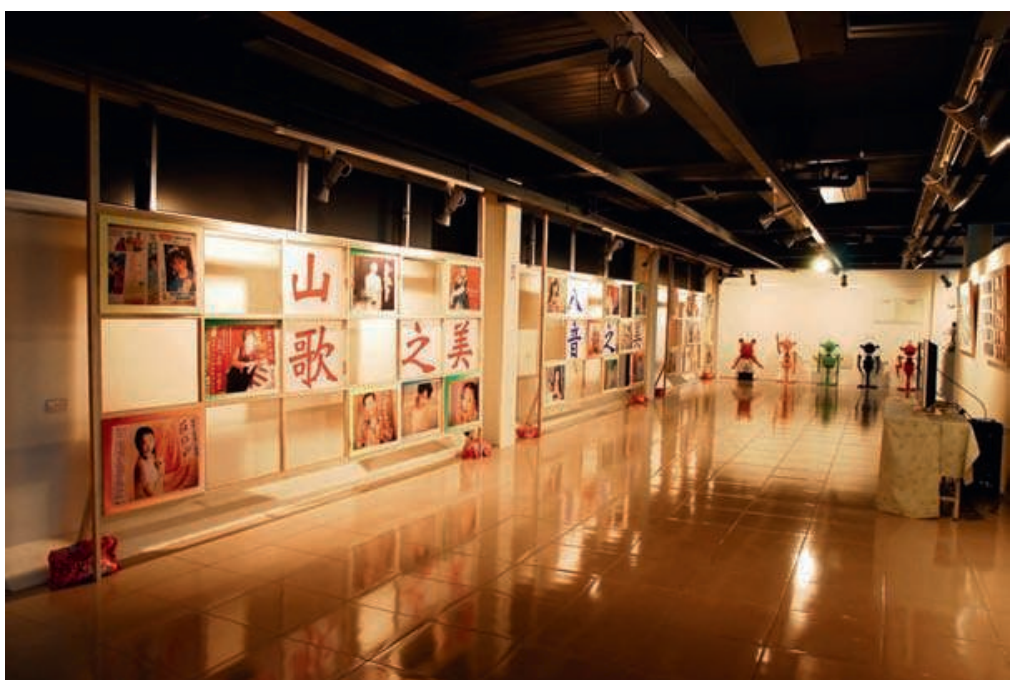

fig. 3

Salle du hall culturel hakka (c) Bureau des affaires hakkas du gouvernement du district de Taoyuan. 
fig. 4

Salle du hall culturel hakka

(c) Bureau des affaires

hakkas du gouvernement

du district de Taoyuan.

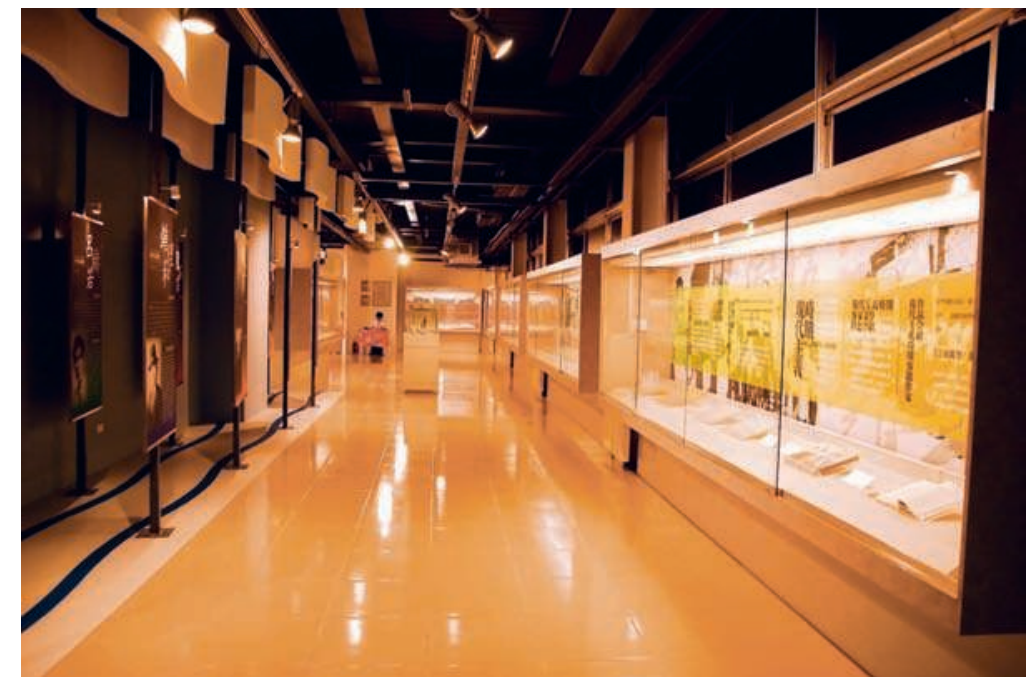

fig. 5

Salle du hall culturel hakka

(C) Bureau des affaires

hakkas du gouvernement

du district de Taoyuan.

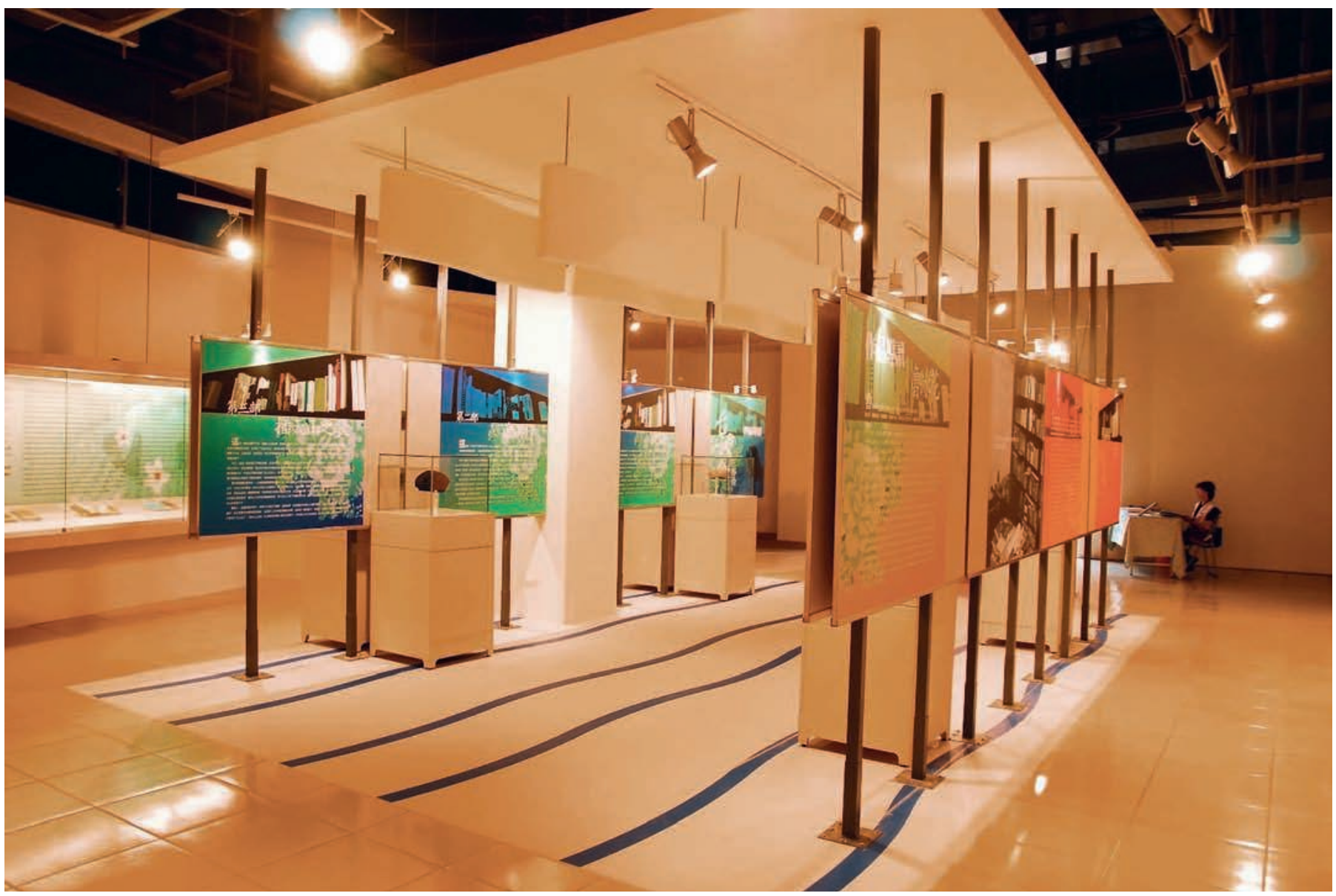




\section{Yingti (硬體) et ruanti (軟體)}

Deux termes sont revenus très régulièrement dans les discours des acteurs impliqués dans le projet: le couple yingti (硬體) et ruanti (軟體). Lors de la présentation que je fis à ce sujet dans un colloque, plusieurs chercheurs taïwanais reconnurent qu'il s'agissait là de catégories couramment employées dans les politiques de la culture, relevant d'une forme d'habitus langagier qui n'avait jamais fait l'objet d'une analyse.

Yingti et ruanti ne renvoient pas à des forces ou à des substances qui seraient pensées comme mutuellement exclusives, mais constituent plutôt une dichotomie agissante, construisant la réalité qu'elles ordonnancent. Ce sont des catégories ontologiques, qui définissent ce qui est, constituent la réalité en la portant à l'être et la classifient. Elles sont à la fois antagonistes et complémentaires, partageant le morphème ti (體). Ti, à la forme nominale, signifie à la fois corps et partie d'un corps, substance et état d'une substance, forme, style et système; à la forme prédicative, il signifie faire l'expérience de quelque chose. Yingti et ruanti ne sont utilisés, en chinois, qu'à la forme nominale; ying et ruan sont des qualificatifs qui désignent deux états possibles d'un ti. Ying est le dur, le rigide, le ferme; ruan est le mou, le flexible, le souple. Ces catégories n'existent pas indépendamment l'une de l'autre, le ruan étant toujours utilisé par contraste avec le ying, et réciproquement.

À la forme adjectivale, ying et ruan sont d'usage courant dans la langue chinoise. Le champ sémantique dans lequel ils sont le plus souvent employés est celui de l'informatique, et la meilleure traduction que l'on peut en donner est anglaise: hardware et software. Yingti est le matériel, ruanti le programme informatique. Dans un pays dont la souveraineté n'est pas reconnue sur la scène internationale, et qui constitue de facto un État, mais un État qui s'affirme en se démarquant de celui qui lui dénie sa souveraineté - l'État chinois -, et qui doit sa renommée mondiale de «dragon», partie prenante du «miracle asiatique» des années 1980, à son industrie tournée vers l'exportation de composants et de produits électroniques, il n'est sans doute pas étonnant que la politique culturelle étatique soit pensée à travers des catégories qui sont celles du monde de l'informatique. Tandis que, dans les années 1990 et 2000 , le voisin chinois devenait l'atelier du monde, l'économie taïwanaise n'a pu survivre qu'en misant sur les hautes technologies et les industries de pointe. L'industrie high-tech est particulièrement concentrée dans la partie nord-ouest de l'île, dans les xian de Hsinzhu et de Taoyuan. L'omniprésence, dans le hall hakka, du multimédia basé sur les dernières technologies et d'écrans plats ultrafins projetant des dessins animés dernière génération en offre une illustration, constitutive de l'image qu'il entend projeter de la modernité hakka et taïwanaise. La muséification de la culture est conçue comme une opération relevant de la promotion de l'industrie culturelle de pointe, au même titre que les autres secteurs de l'économie.

Tantôt les termes yingti et ruanti étaient utilisés séparément, pour distinguer différentes étapes et divers acteurs du processus de construction du hall, tantôt ils se suivaient pour former un morphème et désigner une 
7. www.hakka.gov.tw/ct.asp ?xltem $=41451 \&$ ctNode $=308 \&$ $\mathrm{mp}=307 \& \mathrm{ps}=1$. totalité. Leur usage apparaît comparable à ce que dit Marcel Granet du yin et du yang: «Si l'on tend à les considérer comme des principes efficients, on tend, aussi, simultanément et dans la même mesure à voir en eux des rubriques efficaces. Ils forment à la fois un couple d'activités alternantes et un groupement biparti de formes alternées. Ils président au classement de toutes choses. 》 (Granet 1968 [1934]: 117)

Employés séparément, ying et ruan se succédaient, ying précédant ruan. Ils désignaient alors différentes étapes du processus de construction du hall et divers acteurs dans la division du travail entre le centre et le local. Le centre, zhongyang, l'État, devait financer la construction du bâtiment, alors que le local, le gouvernement du xian, était en charge de la «substance" culturelle. Le président du Keweihui, ministre des affaires hakkas, m'expliqua les étapes d'un projet type: "D'abord il y a le plan pour la construction (jianzhu de guihua) [...] ensuite vient le plan pour le software à l'intérieur (neibu ruanti de guihua). " Dans cette phrase, ruanti est remplacé par neibu («l'intérieur»); yingti n'y figure pas, mais sa présence est implicite par le contraste entre les deux propositions. Cette distinction entre ying et ruan est dupliquée et affinée à l'échelon local du xian. Le bureau de la culture du xian de Taoyuan, le wenhuaju, est divisé en deux équipes, celle qui s'occupe d'organiser des festivals et de sponsoriser des activités culturelles ("les éléments ruanti »), et celle chargée de la conservation et la restauration des bâtiments et monuments historiques (« l'aspect yingti»).

Ces divisions et subdivisions reviendront au fil de la description, mais soulignons d'emblée que ces catégories ne se réduisent pas à une opération de classification en contenant/contenu, construit/non construit, matériel/immatériel; elles revêtent également une dimension temporelle, désignant deux éléments qui se succèdent l'un à l'autre puisqu'ils se définissent mutuellement l'un par rapport à l'autre. C'est pourquoi elles sont pensables comme constitutives du déploiement des politiques culturelles dans leur diachronie. Cette temporalité transparaît dans le cours du processus de création du hall et dans sa double inauguration, celle du yingti en novembre 2005 suivie de celle du ruanti en septembre 2008. Désignant des étapes successives mais indissociables, ces termes se référaient au hall comme un tout: ruan, ying étaient alors accolés dans la même phrase, ruan précédant ying. Par exemple, le président du Keweihui, Huang Zhuwei, lors de l'inauguration du ruanti, s'exclama: «Je m'attendais à visiter un wenziguan [«hall à moustiques»], je suis vraiment agréablement surpris qu'il soit aussi bien! » II ajouta: « II ne faut pas le laisser vide, mais renforcer le ruan-ying $t i{ }^{7}$. » Difficilement traduisible, cette dernière expression souligne que ruan et ying sont les deux aspects complémentaires sur lesquels il faut agir pour parvenir à un résultat satisfaisant, à une totalité complète.

Le couple yingti/ruanti est ainsi apparu, au fil de l'enquête, comme la matrice problématique à partir de laquelle rendre compte de la création du hall, car c'est celle qui habite les acteurs impliqués dans le projet: celle des rapports entre contenant et contenu, fixité et mutabilité, plein et vide, mais aussi «État» et «société civile». 


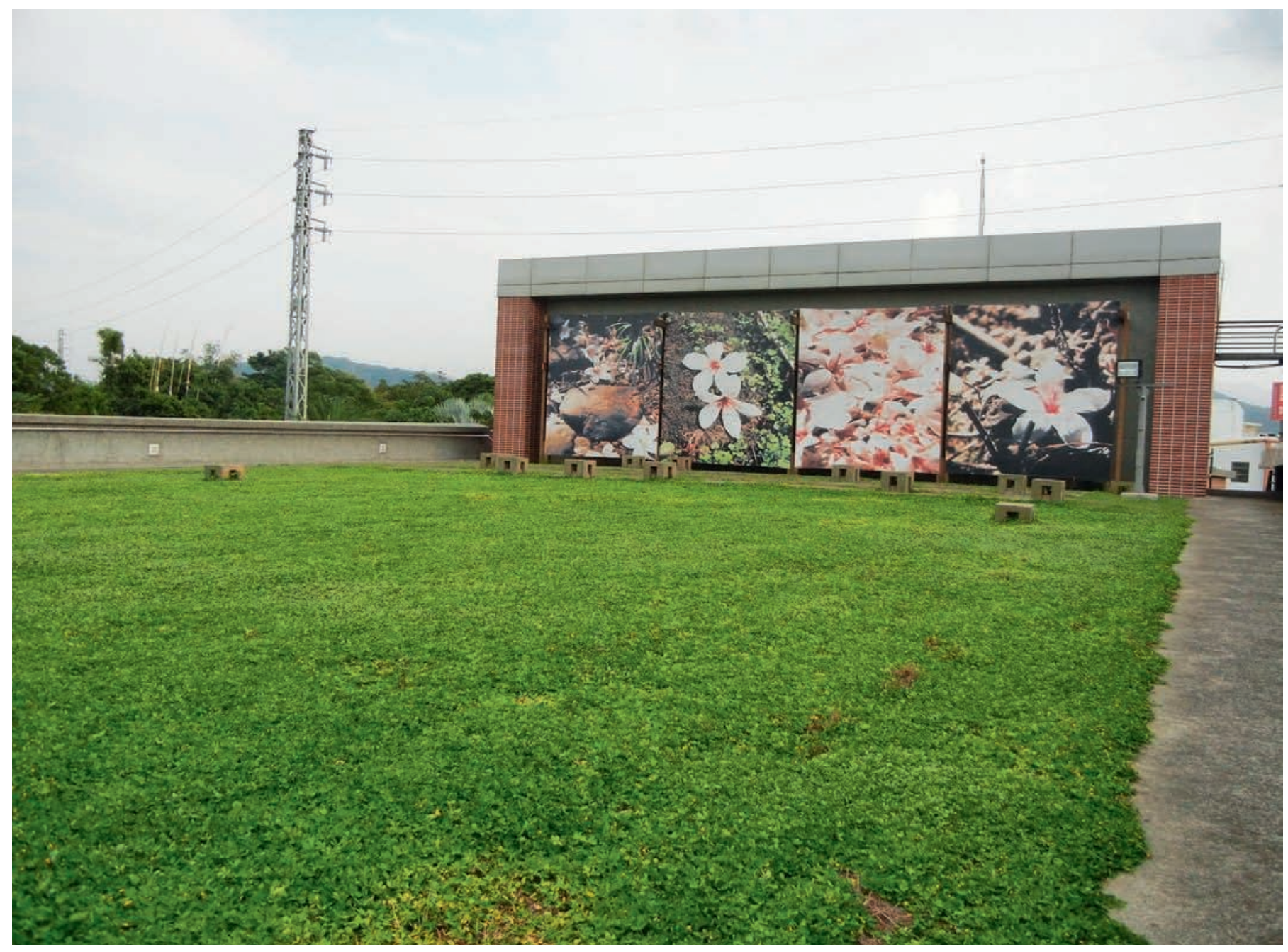

fig. 6

Panneau extérieur

représentant les fleurs

symboliques de la culture

hakka $\odot$ Bureau des affaires

hakkas du gouvernement

du district de Taoyuan. 


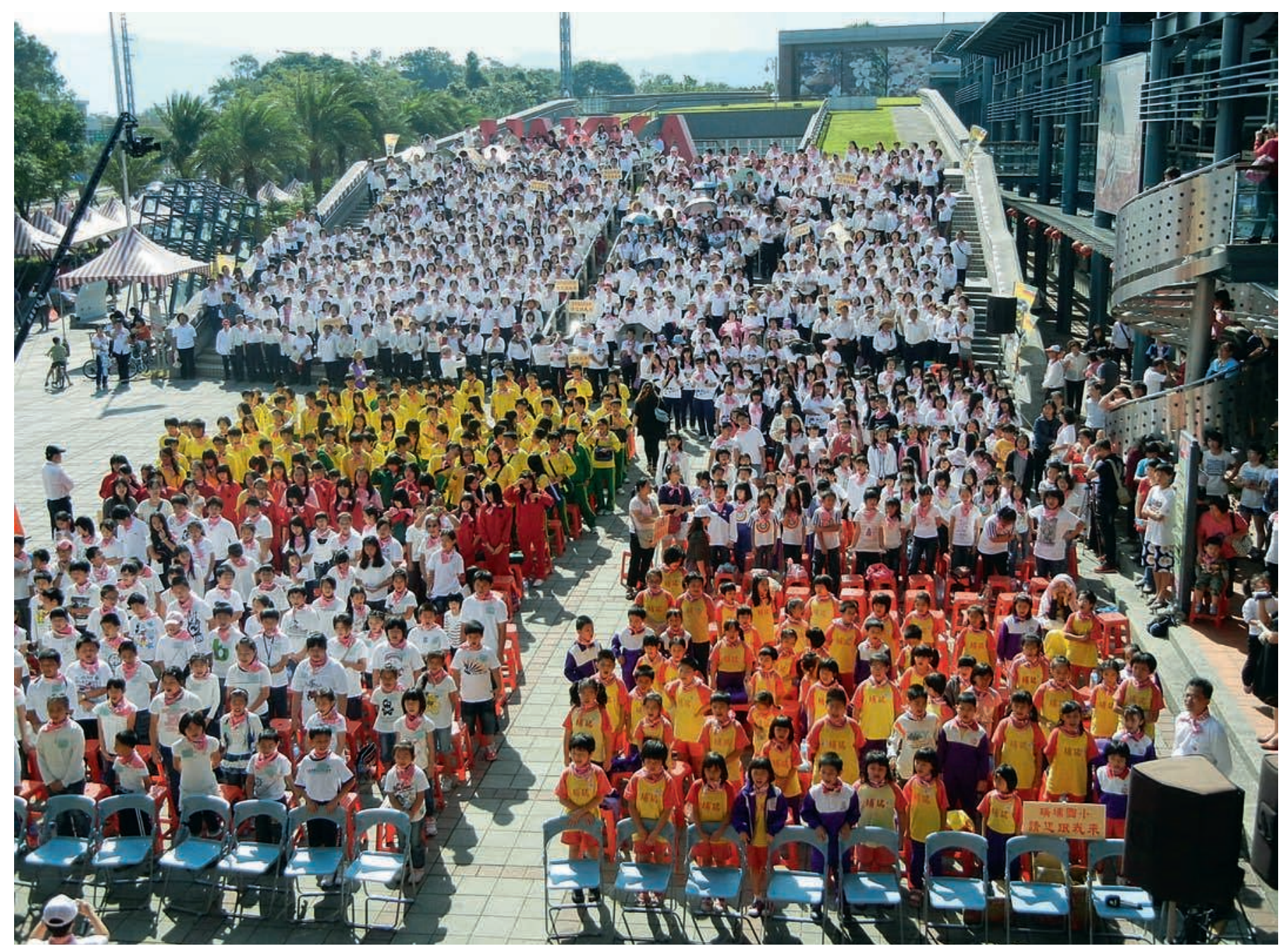

page 130

et ci-dessus

fig. 7

Le Hakka Tung

Blossom Festival

(c) Bureau des affaires

hakkas du gouvernement

du district de Taoyuan.

\section{L'inauguration du yingti}

Le hall culturel hakka du xian de Taoyuan possède la double particularité d'avoir été projeté avant même l'établissement du Comité des affaires hakkas, et d'avoir connu une réalisation difficile et heurtée. D'un coût total de 280 millions de dollars taïwanais, il devait être financé à $50 \%$ par le Comité des affaires hakkas (c'est-à-dire par le gouvernement central) et à $50 \%$ par le xian. La construction commença en novembre 2004, et le bâtiment fut inauguré un an plus tard, le 19 novembre 2005.

Le programme de cette journée comprenait deux parties nettement distinctes. La première, expédiée en moins d'une heure, était la cérémonie de l'«ouverture des portes", jiepai. Elle débuta par le discours des hommes politiques présents: le maire de Longtan, le chef du gouvernement du xian et un membre du Comité des affaires hakkas, représentant respectivement l'échelon du comté (xiang), du district (xian) et du gouvernement central (zhongyang). Ils discoururent en un mandarin ponctué de quelques phrases en hakka. Les responsables politiques à l'échelon supérieur sont majoritairement locuteurs du minnan (ou taïwanais), mais ils tentent toujours 
d'adresser ne serait-ce que quelques phrases en hakka à leur auditoire lorsque celui-ci est, comme dans le cas présent, très majoritairement hakka. Ensuite, tous entonnèrent en chœur, en rang sur la scène aux côtés de personnalités de marque - l'écrivain Zhong Zhao-zheng et quelques chanteurs hakkas de renommée nationale -, l'hymne hakka, Kejiabense. Les stars de la chanson hakka, au premier rang desquelles Lai Bixia, chantèrent ensuite quelques-uns de leurs tubes. Enfin, ce fut le moment de la danse du lion ${ }^{\mathbf{8}}$, et sur fond sonore de percussions la troupe se dirigea vers le côté du bâtiment où elle se posta en bordure de la route pour regarder le chef du xian ôter un grand rectangle de tissu fleuri caractéristique de l'artisanat hakka, découvrant ainsi l'inscription Taoyuanxian Kejia Wenhuaguan. Ainsi se termina, à $10 \mathrm{~h} 30$, le volet officiel des festivités.

Le second volet de la fête consista principalement en des performances collectives, danses ou chants, présentés par des membres d'associations de femmes et des collégiens qui avaient été acheminés jusqu'à Longtan pour l'occasion - et avaient constitué l'essentiel du public de la première partie de la matinée. Les collégiens, revêtus de l'uniforme de leurs établissements respectifs, entonnèrent des chansons apprises en classe de musique. Les femmes, appartenant aux associations locales du xian de Taoyuan, exécutèrent des danses d'une très grande variété. Certaines, arborant un tablier en tissu à fleurs hakka, présentèrent une création chorégraphique inspirée des gestes traditionnels des paysannes hakkas aux champs et au foyer (semailles, moissons, tissage), d'autres, coiffées de chapeaux de cow-boy, entraînèrent le public - et quelques officiels dans un madison ${ }^{9}$.

De l'autre côté de l'escalier en pente douce servant de gradins, dans l'espace ouvert qui devait, plus tard, être aménagé en «jardin écologique», étaient dressés des stands où l'on vendait des snacks. Des candidats aux élections, concurrents du Guomindang (le parti du maire et du chef du xian), y distribuaient leurs tracts sous forme de petits bulletins comportant le nom et le numéro du candidat. On était à une semaine des élections locales du 3 décembre 2005, que le Guomindang remporta largement, reprenant de nombreux xian et villes du nord de l'île au Parti démocratique progressiste (le nord de l'île est traditionnellement plutôt «bleu », Guomindang, alors que le sud est «vert», Minjindang).

L'intérieur du hall contrastait avec l'effervescence qui régnait à l'extérieur, non seulement par son silence, mais surtout par son vide. Seul l'espace dévolu aux expositions temporaires offrait quelque chose à voir aux curieux qui s'y aventuraient. On y trouvait un ensemble de tableaux accompagnés de poèmes disposés sur des disques tressés en osier, composés par un amoureux de la poésie hakka. Celui-ci, ne résidant pas à Longtan, mais chercheur en armement stratégique à l'Institut de technologie militaire, était originaire de Miaoli, le xian voisin. On y trouvait aussi des stands où quelques artisans vendaient leurs productions - calebasses calligraphiées dans un mélange de japonais et de chinois par un monsieur, très âgé, dont c'était le passe-temps, peluches, taies d'oreiller et vêtements pour enfants confectionnés dans un patchwork de tissu fleuri par des femmes originaires d'un autre canton du district. Ces exposants avaient été conviés à l'inaugu-
8. La danse du lion (wushi), à ne pas confondre avec

la danse du dragon, est généralement accomplie lors du Nouvel An chinois, mais aussi à l'occasion de festivals religieux ou de cérémonies d'inauguration. Les deux lions sont formés chacun d'une tête à laquelle est rattaché un corps en tissu. Deux danseurs se trouvent à l'intérieur, l'un tenant la tête, l'autre animant le corps et la queue. Ils exécutent des mouvements d'arts martiaux rythmés par des tambours, des cymbales et un gong.

9. Danse collective consistant à accomplir, en rang, une série de pas basiques accompagnés de déhanchés et de claquements de mains, sur fond de musique ultra-rythmée. 
10. China Times, 26 septembre 2007.

11. Les représentants les plus connus de ce courant sont Chen Yingzhen, Hwang Chun-ming, Wang Zhen-he et Wang Tuo (Yang 1997: 334). ration du hall par l'Association pour la diffusion de la culture hakka, basée à Zhongli et chargée par le gouvernement du xian d'organiser l'inauguration. Ils devaient plier bagages le soir même, après la fin des festivités.

Car ce jour-là, compris-je alors, seul le yingti, le bâtiment, était inauguré. L'ouverture du hall aux visiteurs était programmée pour le printemps suivant, après son «remplissage" par l'entreprise privée Shibaoyule à laquelle le gouvernement avait délégué la gestion du hall. Mettant en avant le manque de crédits et l'insuffisance de personnel au bureau de la culture, le gouvernement du xian avait lancé un appel d'offres, selon la procédure dite «BOT» (en anglais build, operate and transfer). II suivait en cela une orientation qui s'est accentuée à Taïwan durant la décennie 2000, tendant à la multiplication des partenariats public/privé dans le secteur muséal. L'appel d'offres avait été remporté par une entreprise d'entertainment, Shibaoyule, filiale du groupe de médias éditant le China Times, Zhongguo Shibao. Or, peu de temps après la journée des festivités de la fin novembre, le gouvernement du xian apprit que la compagnie se retirait du projet. Shibaoyule rompit son contrat, arguant que le hall ne serait pas suffisamment rentable par manque de visiteurs.

Le hall menaçait alors de devenir ce que les Taïwanais appellent un wenziguan (蚊子館), c'est-à-dire un espace inoccupé où résident uniquement des moustiques (wenzi). Cette expression joue sur l'homophonie avec wenzi (文字), terme qui dénote la culture lettrée, étatique (les wenzi sont les caractères écrits). Près de deux ans plus tard, parut dans le China Times un article intitulé « 31,5 milliards [de dollars taïwanais] pour bâtir 80 résidences à moustiques ", qui affirmait: «Le gouvernement n'a rien fait jusqu’à présent pour corriger le phénomène de wenziguan! Selon un document émis par la commission des comptes du Yuan législatif, au mois d'août de cette année, il y avait 80 installations publiques laissées à l'abandon, dont 40 vides et 40 avec un faible taux de visiteurs 10 [...]. " Les gens de Longtan, non dupes des fins électoralistes de cette manifestation inaugurale, ironisaient, eux aussi, sur ce hall aux dimensions pharaoniques dont les seuls visiteurs seraient, probablement, les moustiques.

\section{La genèse politique du software (ruanti)}

Si l'inauguration du bâtiment était un geste électoral, c'est aussi parce que la genèse du hall était intrinsèquement liée à la mise en place d'un régime démocratique partisan à Taïwan. Dès l'origine, le hall a été conçu autour de deux figures originaires de Longtan, l'écrivain Zhong Zhao-zheng et le compositeur Deng Yu Shian. C'est ce qui apparaît à la lecture des documents produits par le bureau de la culture (wenhuaju) du gouvernement du xian, où ces figures locales sont définies comme «les axes autour desquels s'articule le plan d'exposition du software (zhanshi ruanti guihua)".

Deng Yu Shian était déjà commémoré à Longtan, au centre même du bourg. Une stèle lui est dédiée sur le site du mémorial aménagé sur le toit du bureau municipal du tourisme, sur la rive nord de l'étang de Longtan. L'inscription qui recouvre la stèle relate brièvement sa biographie. Né en 1906 à Longtan dans une famille de lettrés, il a fait ses études à l'École normale de Taipei; il est mort de maladie en 1944. II est présenté comme 
«la fierté de Longtan et le père de la chanson populaire taïwanaise». Une seconde stèle est dédiée à Zuo Naifu, dont il est dit qu'il était un habitant de Longtan, chauffeur de taxi né en 1944 et mort noyé en 1977 en essayant de sauver une femme qui tentait de se suicider dans l'étang. Les deux monuments ont été érigés en 2004 par le maire de Longtan. Outre leur commune origine dans le xiang de Longtan, les deux personnages ont la particularité d'être morts jeunes, et peuvent donc être assimilés aux malemorts qui constituent une grande partie des divinités du panthéon chinois. II s'agit d'êtres humains ayant connu une mort prématurée; le culte qui leur est rendu doit les empêcher de demeurer à l'état d'âmes errantes. Lorsqu'un tel culte est initié, ils sont promus au statut de dieu. C'est à cette élévation, sélective puisqu'elle ne concerne qu'un seul de ces deux malemorts, que nous assistons par le déplacement du lieu de culte d'un espace en plein air, au bord du bassin de Longtan, vers un lieu clos, bâti, celui du hall. Nous sommes en présence d'un de ces «transferts de lieux symboles» (Baptandier 1996: 101) qui sont, en Chine, au principe même de la transposition de la mémoire qu'ils véhiculent.

À l'occasion de cette translation, le chauffeur de taxi, malemort héroïque, fut remplacé par une autre figure locale, bien vivante, l'écrivain Zhong Zhao-zheng. Cet écrivain de renom, habitant Longtan, est à la fois l'un des deux personnages principaux du hall et son véritable initiateur. Né en 1925 dans le village de Jiuzuoliao, tout près de l'endroit où le hall hakka a été édifié, il a reçu une éducation scolaire japonaise. Consacrée à la description du mode de vie paysan des Hakkas de la contrée autour de Longtan, son œuvre s'inscrit dans le mouvement de «littérature du terroir» (xiangtu wenxue). Ce courant apparut dans les années 1960 et 1970 par réaction à la littérature anticommuniste et classique chinoise promue par le parti-État Guomindang. Un ensemble d'écrivains s'attachèrent à dépeindre la vie rurale locale dans un style simple et selon un parti pris réaliste. Sans figurer parmi les plus célèbres, Zhong est néanmoins considéré comme un auteur de premier plan ${ }^{\mathbf{1 1}}$. Son œuvre se trouve dans le corpus littéraire étudié par les écoliers, et tous les Taïwanais connaissent le film qui a été adapté de son premier roman, Lubinghua, dont le générique est l'une des plus célèbres chansons taïwanaises. Le roman décrit l'arrivée d'un professeur de dessin dans un kejiazhuang, durant les années 1960, et son opposition au corps enseignant qui met en application l'idéologie du Guomindang. J'ai rencontré Zhong Zhao-zheng chez lui, dans le centre de Longtan, où il vit au-dessus de la mercerie tenue par sa belle-fille, qui porte le nom éponyme de ce roman.

Zhong est un des adhérents de la première heure du Parti démocratique progressiste (PDP), le Minjindang, autorisé après la levée de la loi martiale, en 1987. C'est durant la décennie suivante, et à partir des activités littéraires et politiques de Zhong, que l'idée d'un hall culturel hakka prend forme. Durant la brève période où Taoyuan est gouverné par une majorité PDP, Zhong fait part à la présidente du xian d'un projet de hall dédié à la culture hakka. II soutient ensuite la campagne du candidat PDP aux premières élections présidentielles démocratiques tenues à Taïwan, en 2000, en présidant un comité de soutien électoral hakka (kejia zhuxuantuan). Après l'élection de Chen Shui-bian, Zhong est nommé parmi

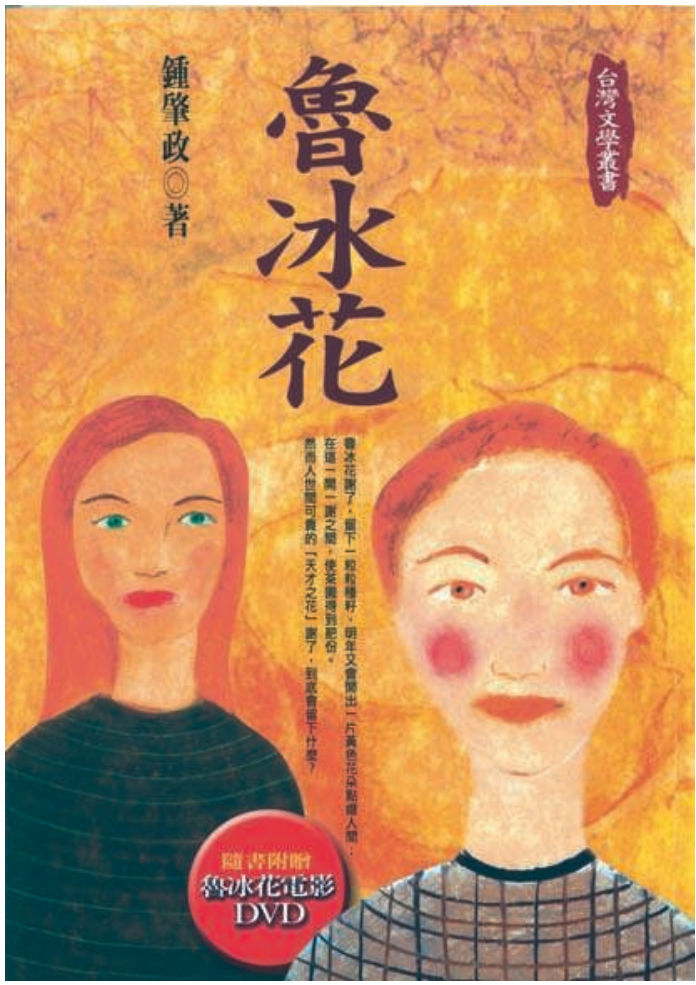

fig. 8

Lubinghua, roman de Zhong Zhao-zheng,

réédition (date inconnue), édition originale de 1979. Collection Li-Chung Jeng. 


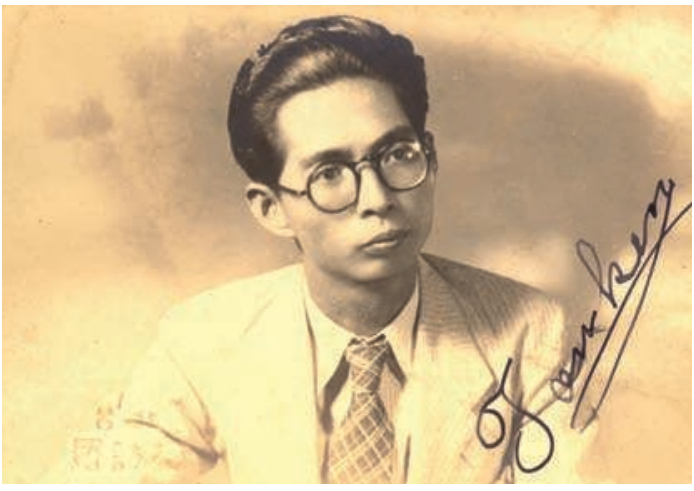

fig. 9

Deng Yu Shian

(C) Succession Deng Yu Shian. les «conseillers spéciaux» de la présidence, poste purement honorifique qui lui permet néanmoins de peser auprès du Keweihui afin d'obtenir des subsides pour le projet. En effet, celui-ci a, entre-temps, été mis entre parenthèses par le gouvernement du xian de Taoyuan, où le Guomindang est redevenu majoritaire. La renommée de Zhong et sa proximité avec le pouvoir central lui permettent de surmonter les problèmes que pose l'adversité des obédiences politiques entre gouvernement central (PDP) d'un côté, xian de Taoyuan et xiang de Longtan (Guomindang) de l'autre.

L'histoire du projet, à ce stade, ne se résume cependant pas à une question de factions politiques. Après 2001, dès lors que le Comité des affaires hakkas décide de faire bâtir un hall, musée ou centre culturel hakka dans chaque xian de Taïwan, les différentes localités du district se livrent une compétition serrée pour accueillir le projet. Alors que le président PDP du Comité des affaires hakkas pesait de tout son poids pour que soit choisie sa localité d'origine, dans l'ouest du xian de Taoyuan, Longtan finit par l'emporter grâce au front commun que formaient l'écrivain Zhong et le maire Guomindang de Longtan. L'appartenance partisane n'a donc pas prévalu. En outre, Longtan se situe dans une région du sud du xian de Taoyuan dont le gouvernement entend faire une région touristique. Le xian de Taoyuan a fait l'objet d'un «zonage», d'un découpage en zones à dominante industrielle, agricole et touristique. Dans ce plan d'ensemble, le comté de Longtan est placé dans la troisième catégorie. Cela tient à ce qu'il comprend en son extrémité sud le réservoir Shimen Shuiku, propriété de l'État taïwanais, un site très visité pour ses panoramas et ses restaurants où l'on sert des poissons d'eau vive, mais aussi quelques villages préservés que le gouvernement du xian entend valoriser, tel Sankang aux ruelles anciennes. Les urbains taïwanais sont friands de ces sites qui donnent un aperçu miniature de ce qu'était Taïwan il y a encore à peine quarante ans. Enfin, un argument financier a joué en faveur de Longtan. Le critère de proximité du futur hall avec les villes de Taipei et de Taoyuan (et donc le public urbain que les concepteurs du projet espéraient attirer) s'avérait difficile à respecter sans faire exploser les coûts, les prix du foncier étant très élevés dans les zones plus urbanisées du xian de Taoyuan. La localité de Longtan l'emporta grâce à deux atouts: d'une part, la proximité de l'autoroute venant de Taipei en passant par Taoyuan, d'autre part, la présence d'un terrain public qu'il ne serait pas nécessaire de racheter à prix d'or.

Le hall constitue ainsi, littéralement, une parcelle de souveraineté en ce qu'il est construit sur un terrain appartenant à l'État central. La translation depuis le lieu de mémoire aménagé par le maire de Longtan sur les rives du bassin vers ce lieu créé de toutes pièces par l'État et le gouvernement du xian est un acte d'incorporation d'une figure locale au sein d'un ensemble national. Le transfert opère un double mouvement, de l'intérieur de la localité (le bassin central qui a donné son nom à Longtan) vers l'extérieur (le terrain en périphérie du bourg), mais aussi de l'extérieur (la stèle en plein air) vers l'intérieur (du hall construit).

Une fois le bâtiment achevé, personne ne voulut en gérer le contenu, une situation que plusieurs personnes rencontrées à Longtan résumaient par l'expression you ren jian, mei ren guan: «ll y a des gens pour construire, 
mais personne pour gérer.» Le président du Keweihui avait annoncé qu'il financerait l'infrastructure, mais qu'il ne dépenserait pas un yuan de plus pour l'aménagement interne du hall. Le gouvernement du xian, quant à lui, fit montre de générosité en s'engageant à financer le projet alors même qu'il avait été initié par la précédente majorité du Parti démocratique progressiste. Il présenta donc la poursuite du projet comme un acte de responsabilité, la tenue des promesses faites par le gouvernement du xian précédent. Après l'échec de la procédure de délégation au secteur privé, il ne put faire autrement que de mettre en place une équipe pour aménager le contenu. On voit néanmoins ici comment les catégorisations yingti et ruanti agissent en retour sur la politique culturelle en délimitant des degrés de contrôle politique sur la culture. Car cette division paraît avoir un double effet: celui de limiter l'intervention de l'État central sur la nature des activités culturelles ou le «contenu» de la culture (software), et celui de renforcer son contrôle sur ce qui est construit «en dur» (hardware) et restera, de ce fait, fixe, par contraste avec le caractère fluctuant, mouvant, de la culture ${ }^{\mathbf{1 2}}$. La division de toute chose en yingti/ruanti délimite ainsi un champ d'action politique différencié. À l'échelon central, l'attribution de la seule construction du bâtiment, à l'échelon du xian, la définition de ce qui doit s'y trouver; le xian lui-même ayant voulu, à l'origine, limiter son intervention aux installations tangibles et déléguer sa gestion (le software) au secteur privé.

\section{L'aménagement du hall:}

\section{l'inscription des Hakkas dans un récit national}

L'inscription des halls dans la grille administrative taïwanaise (un dans chaque xian ou ville) revêt une signification particulière en ce qui concerne les Hakkas. Dans l'espace de la Chine impériale, les particularités culturelles locales ne fonctionnaient pas comme des marqueurs ethniques, mais correspondaient à des délimitations géo-administratives, celles des provinces; elles coexistaient avec l'identification ethnique des sujets de l'empereur, à l'échelon supraprovincial, comme Chinois hans. Les Hakkas occupaient à cet égard une situation particulière puisqu'ils se situaient dans une zone frontalière aux provinces du Guangdong, du Fujian et du Jiangxi. Leur relégation dans les contrées plus montagneuses et moins fertiles, où ils se trouvaient en contact avec des minorités non hans, est au principe même de l'ethnogenèse hakka (Leong 1997). Cette situation périphérique a conduit, au cours de l'histoire chinoise, à l'identification des Hakkas en tant que groupe ethnolinguistique plutôt que comme sous-ensemble provincial ${ }^{13}$. L'absence d'adéquation entre particularité culturelle (hakka) et localisation dans la grille administrative provinciale (il n'y a pas de province hakka) les rapprochait du statut des minorités non hans (Constable [éd.] 1996). Le groupe hakka se distinguait de surcroît par sa pauvreté et sa rusticité, ce qui lui a valu d'être durablement stigmatisé (Cohen 2010).

À Taïwan, après la migration, les Hakkas ont connu une situation similaire; dans le Nord, ils se sont retrouvés à cheval sur trois xian. On peut donc voir dans l'imposition par le pouvoir central d'une série de musées et de halls épousant les contours administratifs plutôt que la géographie du peuplement hakka une manière de les extraire des marges de l'État pour les réinscrire à plein dans la collectivité nationale taïwanaise. Bien entendu, ce découpage administratif sert des fins politiques dans la mesure où
12. Le caractère "sensible» du hardware se mesure également au fait que les associations culturelles locales peuvent, pour ce qui est classifié comme relevan du software, demander des subventions soit auprès du xian, soit auprès du centre, alors que pour ce qui est du hardware, leur demande doit être validée par les deux niveaux.

13. À la différence, par exemple, des Hokkiens du Fujian, des Cantonais du Guangdong, etc. 


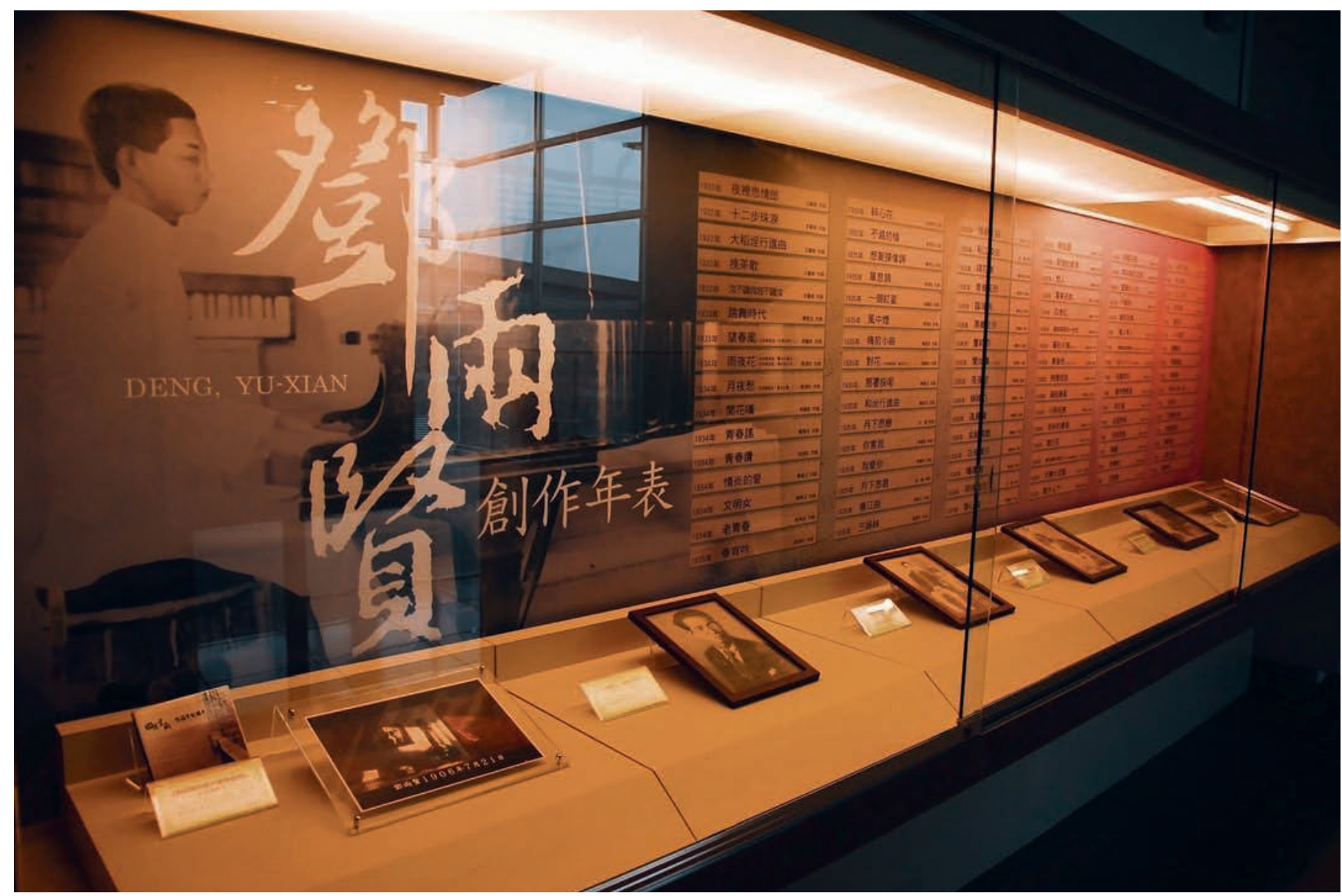

fig. 10

Hall dédié au musicien Deng Yu Shian

(C) Bureau des affaires hakkas du gouvernement du district de Taoyuan. les halls hakkas construits par chaque gouvernement de xian servent de lieu de rassemblement où l'on courtise la clientèle électorale hakka. On peut aussi y voir une tentative de fournir une alternative séculière et administrative à l'activité religieuse qui se déploie au temple des Yimin, non loin de Longtan. Cette communauté de culte transcende les divisions administratives. Les quinze localités hakkas (kejiazhuang) organisent chacune leur tour et chaque année, le vingtième jour du septième mois lunaire, la célébration des Yimin, Yiminjie, dans le temple du même nom. II s'agit de commémorer en la réactualisant l'unité des Hakkas forgée dans les luttes interethniques du temps de l'immigration ${ }^{\mathbf{1 4}}$. S'il existe par ailleurs tout un éventail de temples locaux gérés par les communautés villageoises, la fête des Yimin est bien la principale activité communautaire des Hakkas. Le hall culturel hakka vise à en être l'équivalent, sinon le substitut séculier. Le pouvoir chinois n'a-t-il pas, de tout temps, cherché à placer les dieux locaux dans une hiérarchie administrative céleste agréée par l'État? Seulement ici, dans le Taïwan de la modernité high-tech, ce ne sont pas les Yimin que le pouvoir politique admet dans un cadre officiel, mais des héros de la littérature et de la musique.

Si le bâtiment est un guan, un bâtiment administratif, il s'apparente à un temple séculier. L'espace d'exposition est situé dans le bâtiment principal - le trait central du U - parallèle à la route. Comme un temple, 
il forme un ensemble composite, un assemblage d'unités indépendantes les unes des autres. II est un carré magique, un agencement de cinq halls dont deux sont situés au rez-de-chaussée et trois à l'étage. Comme tout temple, il a été inauguré deux fois, d'abord l'extérieur, puis l'intérieur. Enfin, à l'égal d'un temple, il articule en son sein intérieur et extérieur, les halls étant desservis par des corridors semi-ouverts délimités par des colonnes. On peut accéder directement à chacun d'eux, soit, pour ceux qui se trouvent au rez-de-chaussée, via la cour ou le couloir extérieur, soit, pour ceux qui se situent à l'étage, à partir de la véranda desservie au nord par la passerelle, au sud par un escalier. Au rez-de-chaussée se trouvent un espace d'exposition temporaire et le hall de la littérature; à l'étage, le hall de Deng Yu Shian, le hall de Zhong Zhao-zheng, le hall de la musique, le hall de la littérature et un espace des associations. Un élément graphique tisse un fil à travers le hall, un motif à grandes fleurs typique du style des tissus hakkas. II est projeté sur les grands écrans à cristaux liquides ${ }^{15}$ des panneaux d'affichage placés à l'entrée de chacun des cinq halls; et l'imprimé forme la toile de fond de l'ensemble des éléments exposés dans le hall.

Si Deng Yu Shian et Zhong Zhao-zheng sont mis en exergue dans leurs halls respectifs, ils sont des éléments d'un ensemble dans les halls littéraire et musical. Ils n'y sont pas primi inter pares: ils sont une unité parmi un agrégat, une composante d'une série numérique. Le «hall de la musique hakka», Kejia Yinyueguan, contient dix bannières tendues à la verticale, ornées du même motif floral. Placées côte à côte, elles présentent une courte biographie et les principales œuvres de dix musiciens, dont Deng. Le texte en caractères chinois est précédé du nom romanisé et d'une ligne en anglais. Si tous ont en commun d'être d'origine hakka et nés à Taïwan (plutôt dans le nord de l'île), il s'agit là d'un ensemble plutôt hétéroclite. Lai Bixia vient en premier, et est sans conteste la plus connue. Née en 1932 à Hsinzhu, elle est surnommée la reine du shange. Elle s'est passionnée très tôt pour ce genre particulier, les chants entonnés sur les plantations de thé exprimant le dur labeur des Hakkas, et a poursuivi leur collecte tout au long de sa vie. On trouve également dans ce hall Zheng Rong-xing, le directeur de l'Institut national taïwanais de l'opéra, Hsu Min-heng, le compositeur de Kejia Bense et de Wo shi Kejiaren, hymnes hakkas, mais aussi d'une chanson en mandarin à forte tonalité nationaliste, Song ni yi ba nitu, ou encore Hsieh Yu-wei, le chanteur hakka le plus jeune et le plus connu à l'heure actuelle. Neuf de ces musiciens, encore vivants pour la plupart, partagent un engagement en faveur de la musique hakka par leurs activités de collecte, de compilation, d'enseignement et de diffusion; un engagement de portée nationale qui s'assortit d'une institutionnalisation de la musique hakka.

Deng Yu Shian, quant à lui, s'inscrit dans la période antérieure; il a vécu sous l'ère coloniale japonaise et est mort juste avant la fin de l'occupation. Dans les années 1920, il a séjourné au Japon pour y étudier la musicologie, qui avait subi, bien plus que la musique chinoise, l'influence de la musique occidentale. L'héritage colonial japonais en matière musicale consiste même principalement en l'adoption, à Taïwan, de la musique de style occidental. L'œuvre de Deng porte l'empreinte de cette période durant laquelle de multiples influences, japonaise, occidentale, locale, se mêlèrent pour donner naissance à la musique populaire taïwanaise (Chao 2009).
14. Il s'agit d'un sacrifice en hommage aux morts hakkas dans leurs batailles contre les Hokkiens. Les Yimin étaient des loyalistes qui se constituèrent en milices pour défaire les rebelles hokkiens dressés contre la dynastie des Qing.

15. Les firmes taïwanaises figurent parmi les leaders mondiaux dans le secteur des écrans à cristaux liquides. 
16. www2.tyccc.gov.tw/ hakka/guid1.html (en chinois).

17. En $1945,70 \%$ de la population taïwanaise parlait et écrivait couramment la langue japonaise, et la majorité n'avait aucune maîtrise du mandarin.
Elle porte également la marque du Huangmingua, politique d'assimilation menée par les Japonais, qui s'est traduite notamment par l'interdiction des chants en chinois, qu'il s'agisse du mandarin, du minnan ou du hakka. Deng a même été fortement critiqué, de son vivant, pour avoir transposé une partie de son œuvre en japonais. Mais dans le contexte actuel d'affirmation d'une identité taïwanaise dont la composante japonaise est une des spécificités, cela n'empêche pas Deng d'accéder à une stature nationale. En décembre 2006, le «concert présidentiel», une tradition inventée par le président Lee Teng-hui, premier président natif de l'île, et poursuivie par Chen Shui-bian, a rendu hommage à deux grandes figures de la musique taïwanaise: Chen Da, un musicien minnan du sud de l'île, et Deng Yu Shian, compositeur hakka du nord.

Les ballades que Deng a composées sont surtout chantées en taïwanais, en mandarin et même en japonais plutôt qu'en hakka. Ainsi, l'une de ses chansons les plus célèbres est Yuyehua («Fleurs d'une nuit de pluie»), originellement accompagnée de paroles en taïwanais et interprétée par la chanteuse Chunchun en 1934 (Li 2001). Bien qu'elle ait reçu une déclinaison hakka, Yuyehua a été le plus souvent interprétée en minnan/ taïwanais (par Teresa Teng, Jiang Hui et Placido Domingo lors d'un concert au Japon, et très récemment, dans une version remixée, par le girls band taïwanais SHE). En somme, Deng est un compositeur de mélodies nourries d'influences diverses et ouvertes à toutes les interprétations. L'autre partie du «hall de la musique» présente la tradition musicale hakka plus ancienne au moyen d'un dessin animé. Celui-ci fait défiler les instruments de musique utilisés dans les genres considérés comme spécifiquement hakkas, le bayin et le beiguan hakka. Deng, père de la musique populaire taïwanaise, est donc incorporé, par son inclusion dans le hall du xian de Taoyuan et dans la classe des musiciens hakka, dans un ensemble dont les contours sont à la fois administratifs (le xian de Taoyuan) et ethniques (le groupe hakka).

Contrairement à Deng, qui appartient à une époque antérieure à celle des neuf autres membres du groupe, Zhong est leur contemporain. Le hall de la littérature hakka, Kejia Wenxueguan, comprend, tout comme celui des musiciens, une «zone des écrivains» dont la biographie et les œuvres sont présentées sur des bannières. Notons le décalage entre la visite proposée par le site internet, où l'on ne trouve que dix écrivains, et le hall lui-même, dans lequel quatre ont été ajoutés, au fond de la salle, en une ligne perpendiculaire à celle des dix autres. La série décimale n'est donc respectée que dans la version idéale du hall présentée sur le site ${ }^{16}$. À la différence des musiciens, les écrivains sont présentés comme «multi-hakkas» (duoyuan kejia), cette expression reprenant le duoyuan du «multiculturalisme» (duoyuan wenhua). À une exception près, les dix premiers écrivains sont nés dans des familles hakkas, mais ils ont écrit l'essentiel de leur œuvre en japonais ou en mandarin; tous sont nés avant-guerre et ont appris le mandarin sur le tard ${ }^{17}$. Les plus célèbres sont Li Chiao, d'origine minnan mais considéré comme un Hakka et auteur de la célèbre trilogie Nuit hivernale, et Dupan Fangge, poétesse, auteure de poèmes en japonais, en mandarin et en hakka. Viennent s'y ajouter quatre écrivains plus jeunes, nés après-guerre. 
La mise en série des bannières, alignées dans chacun des halls, produit un effet d'agrégation. Deng et Zhong y deviennent respectivement membres d'une école de musiciens et d'écrivains portée à l'existence dans l'espace même du hall. Ils sont au sein de ces groupes les seuls natifs de Longtan. Un double mouvement de «localisation» et de «nationalisation" s'opère ainsi: nationalisation de figures locales par leur regroupement avec des artistes hakkas venant d'autres parties de l'île (mais surtout du nord) en même temps que localisation de figures nationales - Zhong, qui vit à Longtan, et surtout Deng Yu Shian, qui l'a très tôt quitté et a fini ses jours à Hsinzhu, mais se trouve ainsi ancré dans son lieu de naissance.

\section{Des choix contestés}

Lors de mes rencontres avec les acteurs politiques du projet, mais aussi avec les gens de Longtan et des environs, membres d'associations culturelles hakkas des alentours, des opinions contrastées sont apparues concernant les choix qui ont été faits pour ce hall. Ces divergences portaient tant sur l'aspect extérieur que sur le contenu et la manière dont on peut «objectiver » la culture hakka dans un hall ou un musée. Ou, en d'autres termes, comment fixer le fluide, le ruan.

Au tout début, les concepteurs du projet avaient pensé à deux options: soit une architecture moderne, soit une architecture traditionnelle dans le style des tulou (土樓), forteresses circulaires typiques de la région d'origine des Hakkas taïwanais dans le sud du Fujian et le nord du Guangdong, ou bien des weilongwu (围龙屋), maisons typiquement hakkas nombreuses dans le sud de Taïwan. Pour ce qui était du contenu, ils hésitaient entre la commémoration de personnalités hakkas et de leur œuvre littéraire ou musicale, et l'exposition d'objets associés au mode de vie paysan des Hakkas (outils agricoles, vêtements et accessoires). Dans les deux cas, c'est le premier terme qui l'a emporté. Ces choix expriment d'une part le désir de tourner le dos au passé des Hakkas, au mode de vie agraire, pauvre et perçu comme arriéré qui a longtemps été le leur, d'autre part de couper les ponts avec le continent chinois (et, là encore, avec le passé) pour mettre en avant leur contribution à la modernité taïwanaise. On trouve donc ici une mise en tension entre tradition et modernité, mais aussi entre ce qui est perçu comme étranger et ce qui est vu comme «autochtone », taïwanais (Harrell et Huang 1994: 2). II s'agit en définitive d'une tentative de rendre l'autochtone moderne, par opposition à l'étranger traditionnel - un renversement du rapport à la Chine de l'ère Guomindang.

Cette problématique paraît rejoindre celle qui a travaillé le mouvement hakka, kejia zuqun yundong, divisé entre les «traditionalistes qui mettent l'accent sur les liens intangibles avec le continent chinois afin de construire l'image d'une identité hakka englobante [et] les radicaux [qui] construisent sciemment une identité hakka centrée sur Taïwan [...] " (Martin 1996: 194). Cette opposition ne peut toutefois être réduite à une ligne de clivage partisane. Si le principal promoteur du hall «moderne», l'écrivain Zhong, est sans ambiguïté un fervent partisan du Parti démocratique progressiste, le gouvernement du xian, du parti Guomindang, a lui aussi penché en faveur de l'option moderniste. Ce qui a pu jouer est le fait que le gouvernement 


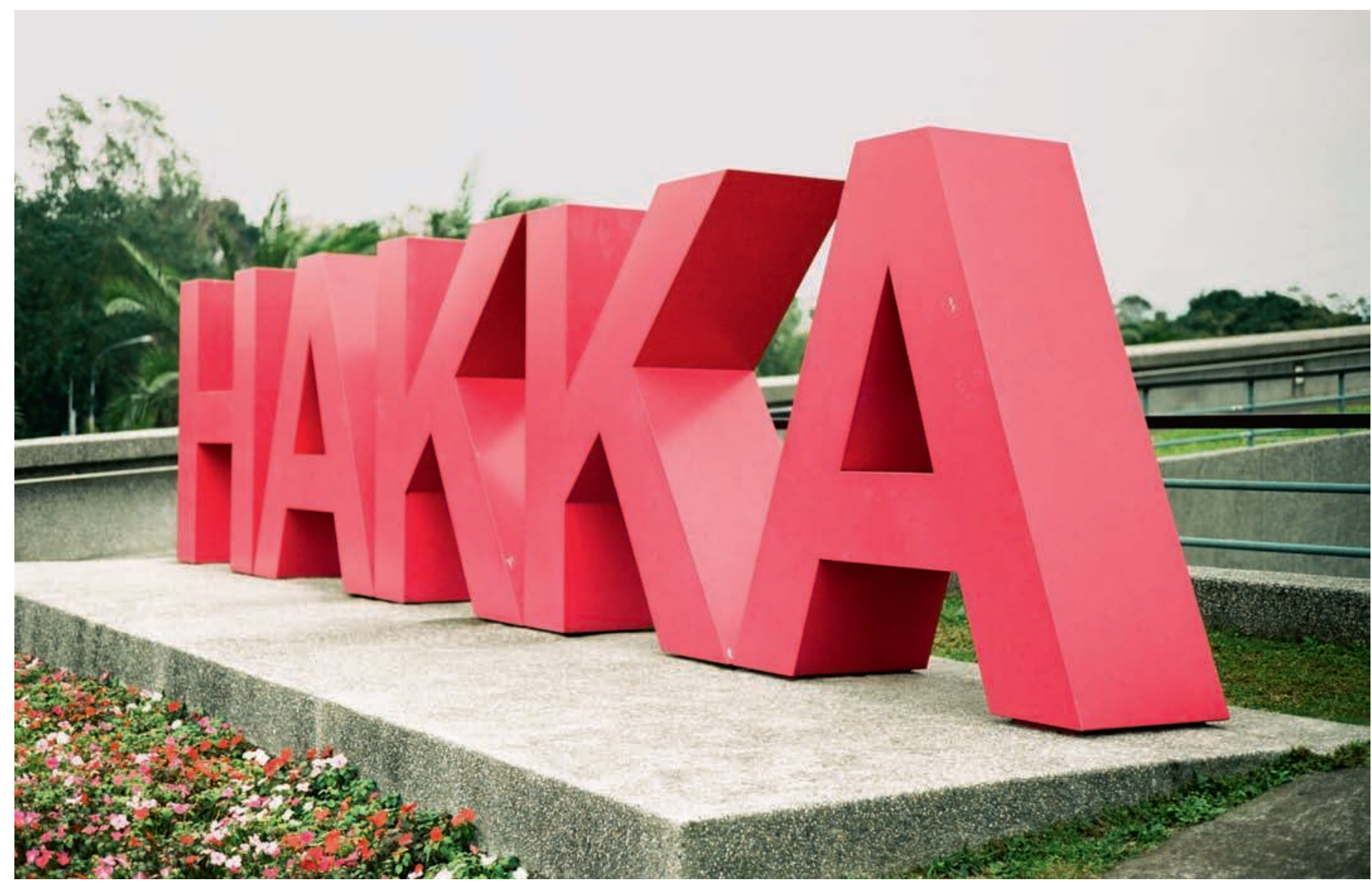

fig. 11

Le logo rose "flashy " du hall culturel hakka de Longtan (Taoyuan)

(C) Bureau des affaires hakkas du gouvernement du district de Taoyuan. précédent avait fait construire, dans Longtan même, un nouveau collège inspiré de l'architecture tulou. En outre, il s'agissait de distinguer le hall hakka de Taoyuan de celui des xian voisins: non loin de là, à Sanhsia, dans le xian de Taipei, le hall hakka est un tulou traditionnel dans une version modernisée; à Longtan, à l'inverse, il s'agit d'un bâtiment moderne avec quelques touches de tradition. Enfin, la personne au sein du bureau de la culture du xian qui venait de prendre sa retraite au moment où je l'ai rencontrée justifiait ce choix par l'impératif de présenter une image plus moderne de la culture hakka et d'aller à l'encontre de leur stigmatisation en tant que paysans pauvres et arriérés.

L'architecture du hall est révélatrice du souci de renvoyer une image moderne. Si son agencement intérieur en cinq parties l'apparente à un temple, il ressemble extérieurement, par sa forme massive, ses lignes rectangulaires et l'omniprésence du verre et de l'acier, à une usine ou un siège social de facture contemporaine. Une petite touche traditionnelle est offerte par la brique rouge orangé qui recouvre la tourelle quadrangulaire à l'avant-poste, et la disposition des bâtiments autour d'une cour peut rappeler les maisons paysannes que l'on trouve encore un peu partout dans les régions rurales. Toutefois, ce matériau signale également l'appartenance du hall à la classe des bâtiments publics: il est utilisé de manière systématique pour les hôtels de police, les hôpitaux et les lycées. La touche ultime a été apportée après ma dernière visite: au sommet de l'escalier, 
visibles de loin lorsqu'on arrive depuis Longtan car perpendiculaires à la route, de grandes lettres roses en écriture latine forment le mot HAKKA le terme d'autodésignation en langue hakka, non transposable en caractères chinois. Leur couleur rose «flashy» renvoie à l'esthétique kawaï importée du Japon, culture jeune très en vogue à Taïwan, tout en rappelant la bigarrure des tissus fleuris hakkas omniprésents dans le hall. Elle s'inscrit également dans la nouvelle politique menée par le xian, qui brandit désormais le mot d'ordre «Fashion Hakka».

Les gens de Longtan, rencontrés alors que le hall était encore vide, se montraient sceptiques quant à son avenir et l'assimilaient à un wenziguan («hall à moustiques»). Ils remarquaient que le hall n'avait rien de distinctement hakka, ironisant sur le fait qu'il ressemblait à une usine. Mais il n'est pas certain qu'un bâtiment plus visiblement hakka les aurait tout à fait comblés. Outre le gaspillage d'argent public dans un bâtiment qui risquait de demeurer sous-employé, ils remettaient en cause la raison d'être du projet lui-même. La plupart d'entre eux étaient impliqués au sein d'associations culturelles actives dans divers festivals de musique ou de théâtre. Mais ils gardaient une certaine réserve vis-à-vis du projet d'un hall uniquement hakka. En effet, ils soulignaient que Longtan avait perdu sa qualité de kejiazhuang, le pourcentage de population d'origine hakka étant descendu à $60 \%$. Par suite de l'installation de waishengren et de minnanren, mais aussi d'aborigènes, la population s'est diversifiée. Malgré les efforts déployés par les concepteurs du hall pour donner une image moderne des Hakkas, les gens de Longtan estimaient que la culture hakka telle qu'ils l'entendaient, la culture traditionnelle paysanne, s'était dissoute dans une modernité non hakka. Ces mêmes personnes étaient pourtant actives dans des associations estampillées "hakkas" ou devant leurs subventions à leurs activités culturelles hakkas. Cependant, parmi la douzaine de dirigeant(e)s d'association rencontré(e)s, deux seulement se consacraient exclusivement à des activités hakkas; il s'agissait dans les deux cas d'associations de niveau xian, c'est-à-dire enregistrées auprès du bureau des associations du xian de Taoyuan. Toutes les autres étaient recentrées sur la localité de Longtan, et leur champ d'activités avait, entre autres, trait à la musique ou à la langue hakka, sans s'y restreindre.

On trouve là tout le paradoxe de la politique multiculturelle menée par le gouvernement taïwanais, pris dans une tension entre la reconnaissance publique donnée à la diversité culturelle et la volonté d'affirmer une unité nationale. L'unité taïwanaise, si elle s'affirme à l'encontre de la culture chinoise, ne peut alors en effet que s'appuyer sur la diversité, et donc sur la revendication de l'appartenance locale (bentuhua) au «terroir » taïwanais (Chun 2002). Les comités ministériels de la culture et des affaires hakkas ont infléchi, au cours des années 2000 , leur politique vers une promotion plus affirmée de la «localité».

\section{Conclusion}

Les catégories à travers lesquelles ce projet de hall a été pensé, yingti et ruanti, forment un couple de "rubriques maîtresses", d'emblèmes à la forte puissance suggestive qui agissent en retour sur le politique dans ses relations avec la culture. La catégorisation yingti/ruanti semble fixer le 
périmètre et les bornes de l'action étatique en matière de culture puisque l'État central se contente de fixer le cadre, le contenant, le construit. Elle a ceci d'intéressant qu'elle émane du centre, des concepteurs des politiques culturelles; elle relève du registre administratif et technocratique dans lequel s'énonce la politique culturelle. Elle est le langage de l'intervention de l'État dans la culture, mais aussi celui par lequel celui-ci fixe ses propres limites par rapport à la culture, ou plus précisément par rapport au ruan, sa part vive, féconde. C'est dans ces écarts entre yingti et ruanti, entre retrait et présence de l'État, que se loge la tension sous-jacente au processus de construction du hall hakka, et que se formulaient les jugements sceptiques des habitants de Longtan et des visiteurs sur le «vide» du hall, lequel est, en fin de compte, un cadre exigeant d'être rempli, mais risquant toujours de ne pas l'être.

Nous avons vu se mettre en place une série d'oppositions entre le projet de hall tel qu'il est impulsé «d'en haut», par l'État, et tel qu'il est mis en œuvre localement; entre son usage à des fins électoralistes et le scepticisme des gens de Longtan vis-à-vis du «hall à moustiques», entre l'imposition d'une grille administrative et la vie culturelle inscrite dans la localité. Mais, plutôt que comme une opposition exclusive, la distinction yingti/ruanti peut s'entendre comme un écho à la distinction entre l'État et la société civile (minjian); une relation de complémentarité antagoniste. C'est au nom de l'autonomie culturelle de la société civile que l'État central a conduit, à partir des années 1990, la privatisation de bon nombre d'institutions. Ainsi, en même temps que le centre impulsait la construction de musées et de halls culturels, s'affirmait la nécessité d'en laisser la gestion du contenu à la société civile. La relation entre yingti et ruanti semble donc recouper, d'une part, celle entre l'État, le central, le public et le permanent (le bâtiment construit sur un terrain public et qui demeure propriété de l'État), d'autre part celle de la société civile, du local, avec le privé, le changeant. Le couple yingti/ruanti, contenant/contenu, dur/mou, fixe/fluctuant, entretient la même relation double que le couple État/société; c'est-à-dire à la fois une relation syntagmatique ou inclusive, suivant laquelle l'État contient la société et la «confirme» (Trouillot 2001: 127), et une relation paradigmatique ou exclusive, suivant laquelle l'État transcende la société. Le premier terme de la relation est donc défini par son opposition au second, tout en l'incluant.

Cette double relation rend également compte du changement de configuration entre État et société qu'a connu Taïwan à la fin des années 1980. On est alors passé d'une situation historique où un État importé de Chine continentale, perçu comme extérieur à la société, jouait le rôle de contenant social, politique et culturel, à une autre où l'État tente de se redéfinir comme endogène, taïwanais, tout en restreignant son intervention dans le domaine de la culture. Cette transition est toujours en cours, mais, comme le souligne Lee Wei-yi dans ce numéro, la restriction du champ d'intervention étatique n'est que partielle, le musée étant toujours le véhicule de l'affirmation d'une revendication politique nationale.

Tout en renonçant à la politique de contrôle et d'homogénéisation nationale au profit de la valorisation des «couleurs locales» de la société 
civile, l'État a cherché à se rendre davantage présent dans la société civile en créant des relais pour se déployer au niveau local (Mengin 2007; Harrell 1996) - le musée local en est une instanciation. La désignation moqueuse des musées locaux construits en trop grand nombre et sans plan de gestion durable comme des wenziguan («halls à moustiques») souligne néanmoins la distance persistante entre la politique muséale étatique et la société locale. Conscientes du problème, les autorités taïwanaises tentent d'y remédier. En 2008, un nouveau plan gouvernemental a ainsi introduit le concept de «cercle de vie culturelle locale» (地方文化生活倦), qui doit permettre d'accroître la participation de la population et de faire du

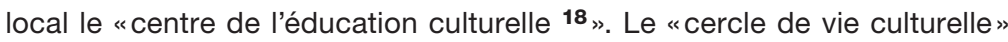
est défini comme étant «composé du hardware et du software (jiang ge lei ruan, yingti zhenghe cheng «difang wenhua shenghuotuan»).

Université de Lausanne Netherlands Institute for Advanced Study Anne-Christine.Tremon@unil.ch
18. Site internet du ministère de la Culture taïwanais, http://www.cca. gov.tw/business.do? method=list\&id=3 


\section{Baptandier, Brigitte}

1996 «Pratiques de la mémoire en Chine. Le dieu des murs et des fossés de Puxi et Hanjiang ", Genèses 23: 100-124.

\section{Bennett, David (Éd.)}

1998 Multicultural States, Rethinking Difference and Identity. Londres, Routledge, 1998.

\section{Chao, Hui-Hsuan}

2009 Musical Taiwan under Japanese Colonial Rule: a Historical and Ethnomusicological Interpretation. Ann Arbor, University of Michigan, thèse de doctorat.

\section{Chun, Allen}

1994 «From nationalism to nationalizing: Cultural imagination and State formation in postwar Taiwan ", Australian Journal of Chinese Affairs 30: 49-69.

1998 «The culture industry as national enterprise: The politics of heritage in contemporary Taiwan ", in Virginia R. Dominguez et David Y.H. Wu (éd.), From Beijing to Port Moresby; the Politics of National Identity in Cultural Policies. Amsterdam, Gordon and Breach.

\section{2 "The coming crisis} of multiculturalism in "transnational" Taiwan ", Social Analysis 46(2): 102-122.

\section{Chung, Yupin}

2005 «The impact of privatization on museum admission charges: A case study from Taiwan ", International Journal of Arts Management 7(2): 27-38.

\section{Cohen, Myron L.}

1996 "The Hakka or "Guest People", dialect as a sociocultural variable in Southeast China ", in Nicole Constable (éd.), Guest People, Hakka Identity in China and Abroa. Seattle, University of Washington Press.

2010 "Configuring Hakka identity and ethnicity, as seen in Meinong, Taiwan, 1963-2008 ", in Zhuang Ying-zhang et Meiling Jian (éd.), Formation and transformation of the Hakka. Jiaotong University Press, (莊英章, 簡美玲 (編者) 客 家的形成與變遷, 交通大學出版 社): 1003-1025.

\section{Constable, Nicole (éd.)}

1996 Guest People, Hakka Identity in China and Abroad. Seattle, University of Washington Press.

\section{Corcuff, Stéphane (éd.)}

2002 Memories of the Future National Identity Issues and the Search for a New Taiwan. Londres, M.E. Sharpe.

\section{Gold, Thomas B.}

1994 " Civil society and Taiwan's quest for identity ", in Stevan Harrell et Huang Chün-chieh, Cultural Change in Postwar Taiwan.

San Francisco et Oxford, Westview Press: 47-68.

\section{Granet, Marcel}

1968 [1934] La Pensée chinoise, Paris, Albin Michel.

\section{Harrell, Stevan et Huang, Chün-Chieh}

1994 "Introduction: Change and contention in Taiwan's cultural scene ", in Stevan Harrell et Huang Chün-chieh (éd.), Cultural Change in Postwar Taiwan. San Francisco et Oxford, Westview Press: 1-18.

\section{Harrell, Stevan}

1996 "Introduction », in Melissa J. Brown (éd.), Negotiating Ethnicities in China and Taiwan. Berkeley, Institute of East Asian Studies, University of California.

\section{Lee, Wei-Chin}

2005 "Taiwan's cultural reconstruction movement: Identity politics and collective action since 2000 ", Issues \& Studies Select Papers from the 2004 Annual Meeting of the APSA Conference Group on Taiwan Studies (CGOTS) 41(1): $1-52$.

\section{Leong, Sow-Theng}

1997 Migration and Ethnicity in Chinese History, Hakkas,

Pengmin, and their neighbors, Tim Wright (éd. posth.). Stanford, Stanford University Press.

\section{Li, Ying-Hui}

2001 "Quatre, lune, espoir, pluie - Deng Yu Shian ” (李映慧 “四、月、望、雨一邓雨贤”, 国立编译馆). Taipei, Institut national de compilation et de traduction.

\section{Lin, Yung-Neng}

2006 "Leisure: a function of museums? The Taiwan perspective", Museum Management and Curatorship 21: 302-316.

\section{Martin, Howard J.}

1996 « The Hakka ethnic movement in Taiwan, 1986-1991 ", in Nicole Constable (éd.), Guest People, Hakka Identity in China and Abroad.

Seattle, University of Washington Press.

\section{Mengin, Françoise}

2007 «A Tocquevillian process. Taiwan's democratization and its paradoxical foundations", in Robert Ash et J. Megan Greene (éd.), Taiwan in the 215t Century: Aspects and limitations

of a Development Model, Londres et New York, Routledge : 232-248.

\section{Shih, Chih-yu}

2002 Negotiating Ethnicity in China. Citizenship as a Response to the State. Londres, Routledge.

\section{Trouillot, Michel-Rolph}

2001 « The anthropology of the state in the Age of globalization. Close encounters of the deceptive kind ", Current Anthropology 42(1): 125-138.

\section{Winckler, Edwin A.}

1994 «Cultural policy on postwar Taiwan ", in Stevan Harrell et Chün-chieh Huang (éd.), Cultural Change in Postwar Taiwan. San Francisco et Oxford, Westview Press: 19-21.

\section{Xiao, Xinhuang et He, Mingxiu}

2006 Taiwan Juanzhi, vol. IX Sihuizhi, Shihuiyundongpian, Nantoushe Taiwan wenxianguan.

\section{Yang, Bi-chuan}

1997 Taiwan lishi ciqu (台湾历史词曲, 台北：前衛॰). Taipei, Qianwei.

ci-contre

Le Hakka Tung Blossom Festival (C) Bureau des affaires hakkas du gouvernement du district de Taoyuan. 


\section{1}

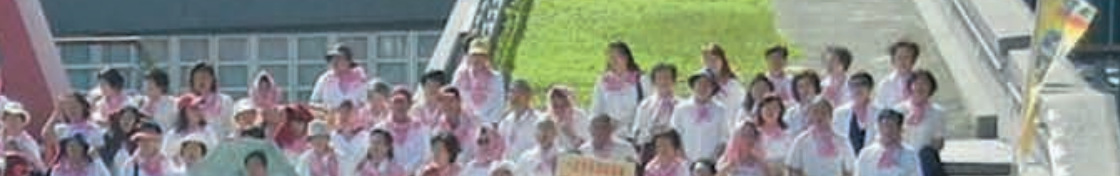
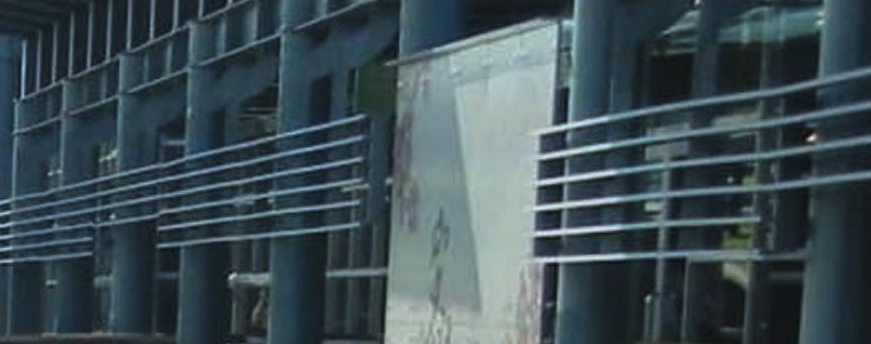
20.

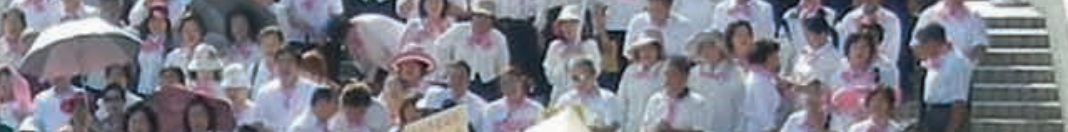

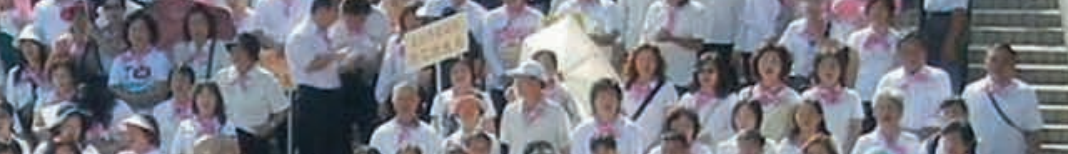
25

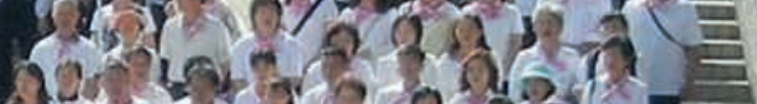

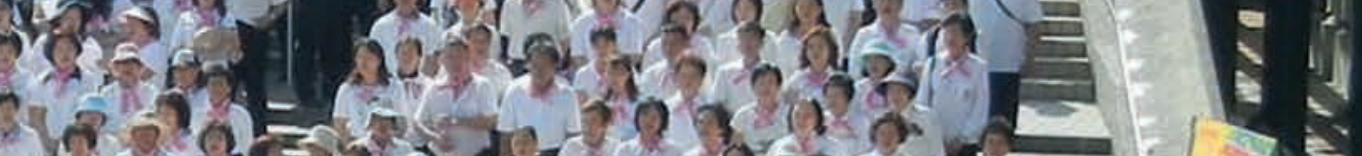

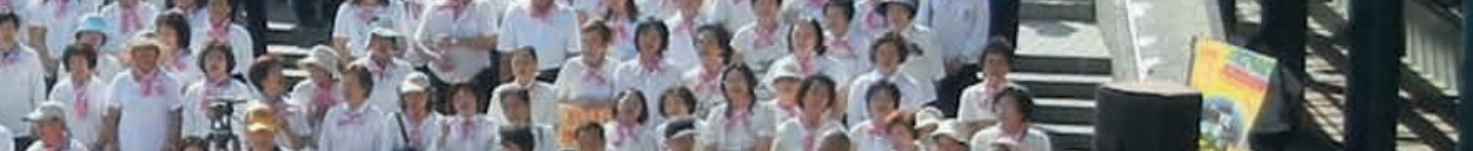

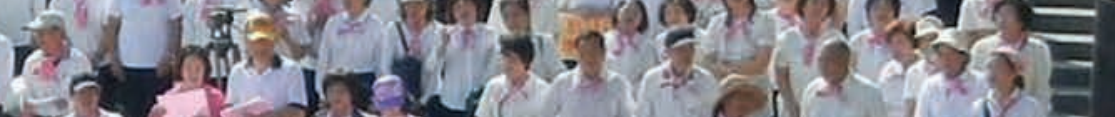

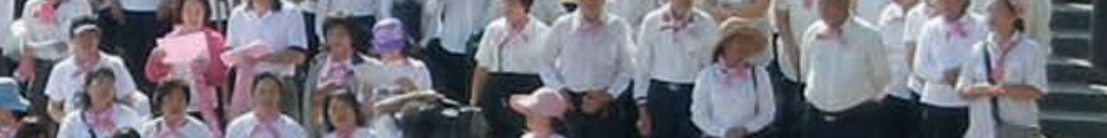

Hof

$10=x^{*}$

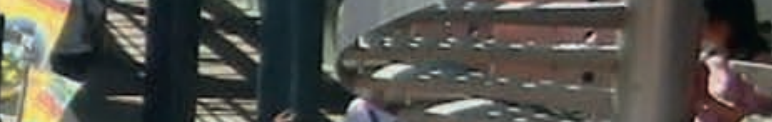

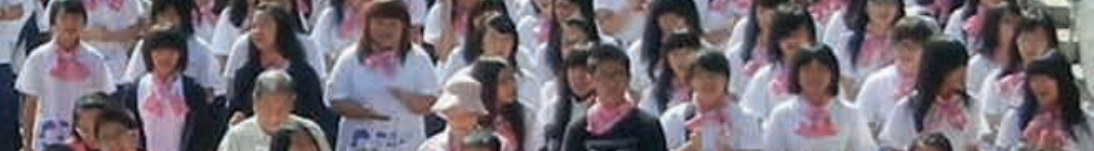

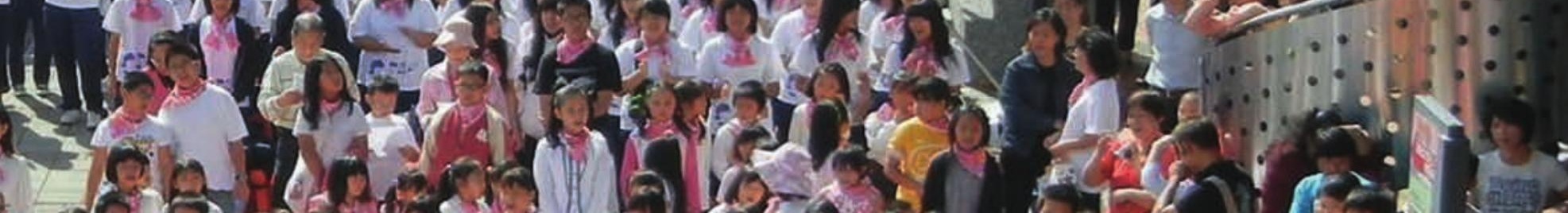

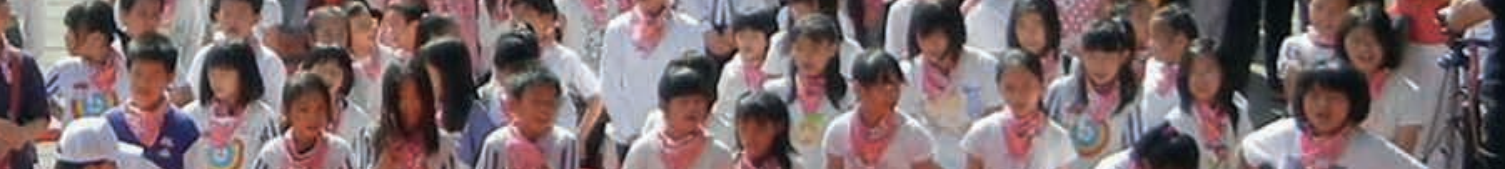

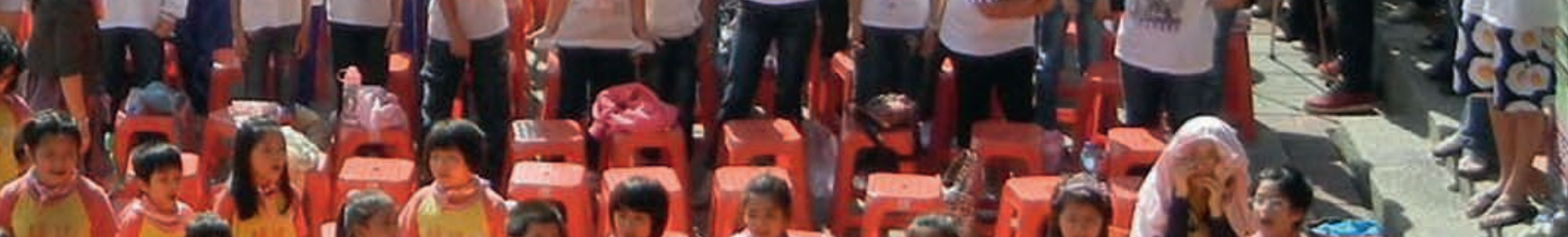

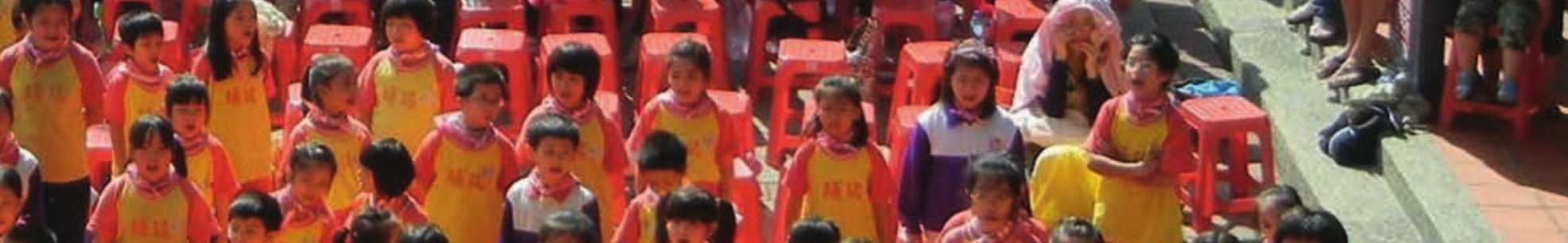

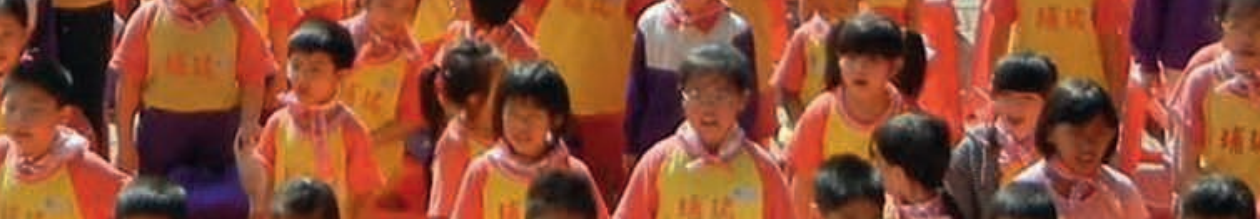

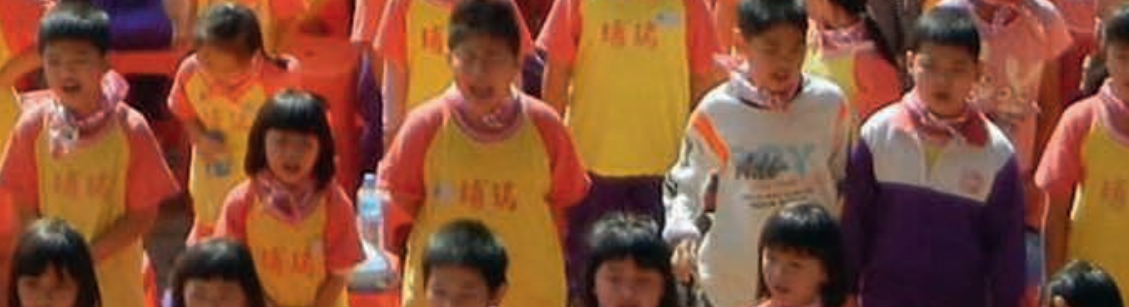

i) 